\title{
Overview of Regioselective and Stereoselective Catalytic Hydroboration of Alkynes
}

\author{
Supriya Rej, ${ }^{\text {a* }}$ Amrita Das, ${ }^{\text {b* }}$ and Tarun K. Panda ${ }^{c *}$
}

\begin{abstract}
Hydroboration of alkynes is of special interest to researchers since it is the most straightforward process for the synthesis of highly important vinyl borane synthetic intermediate compounds. It is significant in terms of both regioselectivity and stereoselectivity, since several positional isomers are formed during the process of hydroboration. Given the importance of this class of compounds, an extensive study has been carried out regarding catalytic condition development and detailed mechanistic studies for selectively obtaining a single isomer. This elaborate review comprehensively summarizes both the regioselective and stereoselective catalytic hydroboration of alkynes. It is aimed at giving readers accurate knowledge of the catalytic systems available for the hydroboration of certain alkynes. We anticipate that this inclusive review will encourage researchers to explore more new types of catalytic systems to achieve the remarkable process of hydroboration of alkynes.
\end{abstract}
1. Introduction
2. syn-Hydroboration of alkynes
2.1. Transition metal-catalyzed syn-hydroboration
2.2. Alkali and alkaline earth metal-catalyzed syn- hydroboration
2.3. Aluminum-catalyzed syn-hydroboration
2.4. Metal-free catalytic syn-hydroboration
3. anti-Hydroboration of alkynes
3.1. Transition metal-catalyzed anti-hydroboration
3.2. Metal-free catalytic anti-hydroboration
4. $\alpha$-Selective hydroboration of terminal alkynes
5. Conclusion and future direction

Keywords: Hydroboration; Alkynes; Regioselectivity; Stereoselectivity; Mechanistic overview

\section{Introduction}

Synthetically important organoborane reagents are highly popular due to their unique reactivity in various synthetic processes. They have been used for several years as synthetic intermediates in the introduction of various functional groups and carbon-carbon bondforming processes. Well-known examples of this are the Suzuki-Miyaura coupling and Petasis boronoMannich reaction. ${ }^{[1-3]}$ The most straightforward strategy used to produce an organoboron compound is the hydroboration of unsaturated organic compounds. This calls to mind Brown and Rao's revolutionary discovery in 1956 of the hydroboration of an alkene moiety using sodium borohydride in the presence of aluminum chloride (Scheme 1a). ${ }^{[4,5]}$ Two years after this discovery, R. Köster reported the hydroboration of alkenes using an alkyl borane reagent (Scheme 1b). ${ }^{[6]}$ These landmark discoveries opened the door for researchers to access synthetically important organoborane intermediates and to use them in several important organic transformations. Given the significance of the hydroboration reaction, various unsaturated functionalities, such as alkenes, alkynes, carbonyls, imines, and nitriles, have been hydroborated over the years. ${ }^{[7-24]}$ Among these, alkyne hydroboration is of special interest as it enables researchers to achieve synthetically important alkenylborane compounds. ${ }^{[25,26]}$ In this regard, pioneering work was done by Brown and Gupta in the hydroboration of alkynes with readily available reactive catecholborane, as reported in $1975 .^{[27]}$






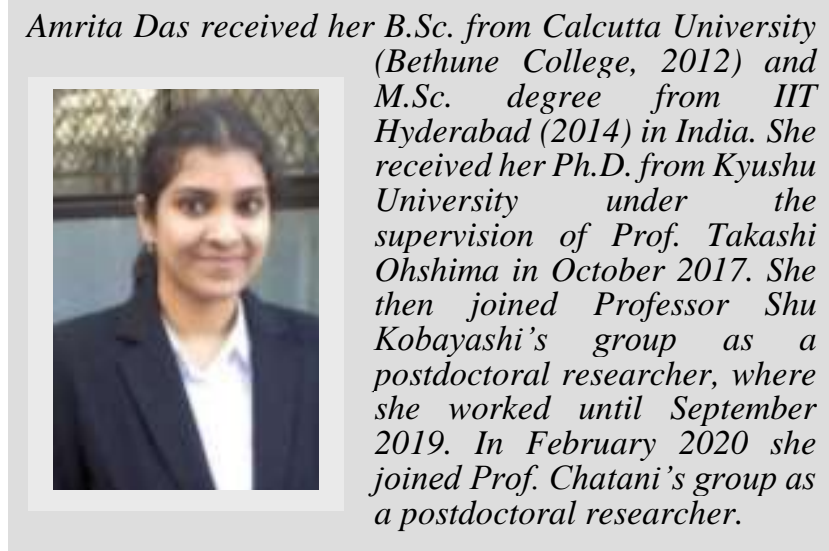

In the early days of research, conventional hydroboration reactions proceeded in a catalyst-free manner, through the addition of diborane $\left(\mathrm{B}_{2} \mathrm{H}_{6}\right)$, borane trihydride complexes $\left(\mathrm{BH}_{3} \cdot \mathrm{L} ; \mathrm{L}=\right.$ stabilizing adducts), or alkylboranes to unsaturated compounds. However, a major concern associated with this protocol was the lack of stability, high air sensitivity, and incompatibility with chromatographic purification of the resulting borane compounds, which limited the practical use of this process in organic synthesis. To address this issue, milder and air-stable dialkoxyborane compounds (HBpin: pinacolborane and HBcat: catecholborane) were introduced. Due to the reduction in electrophilicity, these dialkoxyborane compounds do not undergo catalyst-free transformations unless subjected to very high temperatures. In terms of a catalytic hydroboration reaction, Männig and Nöth first reported the $\mathrm{Rh}$ catalyzed hydroboration of 1-hexyne using HBcat as the hydroboration reagent (Scheme 1c). ${ }^{[28]}$ Subsequently, with the efficacy of the catalytic hydroboration reaction as well as the high stability and ease of purification of alkenylboronates being established, hydroboration of alkynes saw considerable progress, with various catalytic systems such as transition metals, base metals, and Lewis-acids or bases being used.

Tarun K. Panda obtained his M.Sc. from IIT, Kanpur in 2002 and received his Dr. rer. Nat. from Freie Universität Ponet Berlin, Germany, in 2005 under the supervision of Prof. Dr. Peter W. Roesky. In 2006, he joined the working group of Prof. Dr. Matthias Tamm at TU Braunschweig, Germany, as a postdoctoral fellow. He was awarded the JSPS postdoctoral fellowship in 2008 to work with Prof. Kazushi Mashima in Osaka University, Japan. In 2010, he returned to India to join the Department of Chemistry, IIT Hyderabad, India as an Assistant Professor. He was promoted to Associate Professor in 2015 and since May 2019, he has held the position of Professor at the same Institute. His principal research interests include the development of well-defined earthabundant, non-toxic, and environmentally benign metal complexes using non-cyclopentadienyl-based ligands and their exploitation in homogeneous catalysis under ambient reaction conditions.

(a) Early discovery on hydroboration using $\mathrm{NaBH}_{4}$ and $\mathrm{AlCl}_{3}$ (Brown, 1956)

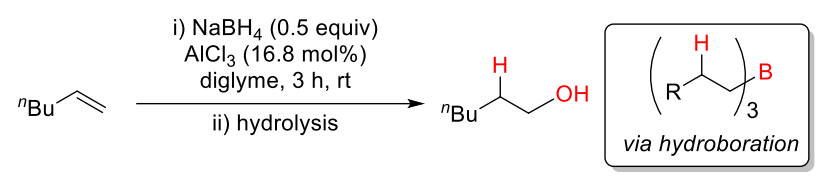

(b) Early discovery on hydroboration using trialkylborane (Koster, 1958)

$$
\stackrel{\mathrm{B}\left(\text { iso- } \mathrm{C}_{4} \mathrm{H}_{9}\right)_{3}}{\stackrel{\mathrm{CH}_{3}}{\longrightarrow}}\left(\mathrm{H}_{3} \mathrm{C}+{ }^{\mathrm{H}}\right)_{3}^{\mathrm{B}}
$$

(c) Transition metal catalyzed hydroboration of alkyne (Noth, 1985)

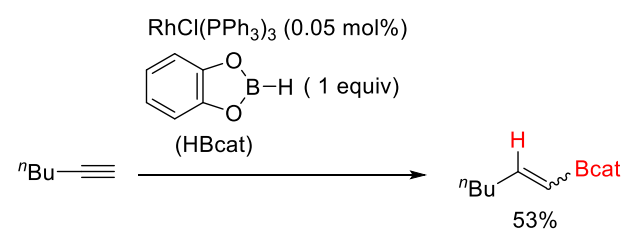

Scheme 1. Early discoveries of hydroboration reaction using (a) sodium borohydride and (b) trialkylborane. (c) Early report of transition metal-catalyzed hydroboration of alkynes using HBcat.

The ability to control the regioselective and stereoselective orientation of afforded alkenylboranes places catalytic hydroboration of alkynes in the limelight. From a stereoselective point of view, hydroboration of alkynes can proceed in two ways, via $s y n$-addition or anti-addition, affording either syn1/syn-2 or anti-1/anti-2 respectively (Scheme 2a). Regioselectivity of the hydroboration of alkynes can also yield two forms of products, namely, $\alpha$-selective borylated product $\boldsymbol{\alpha - 1 / \alpha - 2}$ or $\beta$-selective borylated product $\boldsymbol{\beta}-\mathbf{1} / \boldsymbol{\beta}-\mathbf{2}$ (Scheme $2 \mathrm{~b}$ ). Regioselectivity and stereoselectivity of hydroboration of alkynes largely depends on the type of catalyst used. A well-known mechanism for achieving transition metal-catalyzed $s y n$-hydroboration using HBpin is the generation of metal hydride and subsequent syn-hydrometallation. Conversely, the mechanism for obtaining antihydroboration often involves multiple steps. Notably, anti-hydroboration is uncommon and often difficult to control due to the isomerization process. Overall, synand anti-hydroboration mechanisms rely largely on catalysts. Keeping in mind the significance of this, in this review, we summarize the regio- and stereospecific hydroboration of alkynes. We believe that this overview will enable readers to explore the topic further, gaining greater insight into the mechanistic aspects of the specific hydroboration of alkynes. 
(a) Stereoselective hydroboration of internal and terminal alkyne

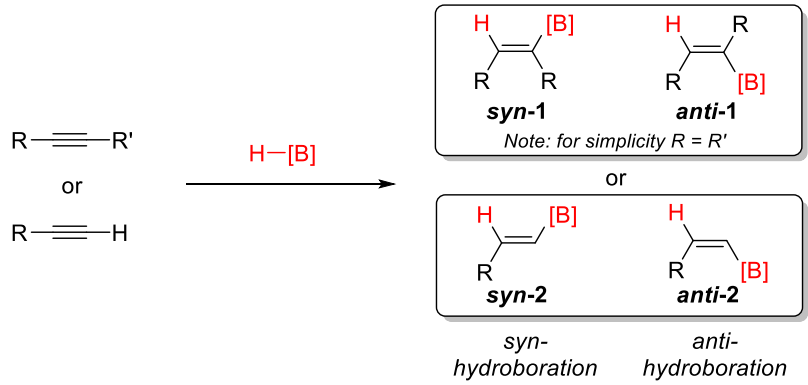

(b) Regioselective hydroboration of internal and terminal alkyne

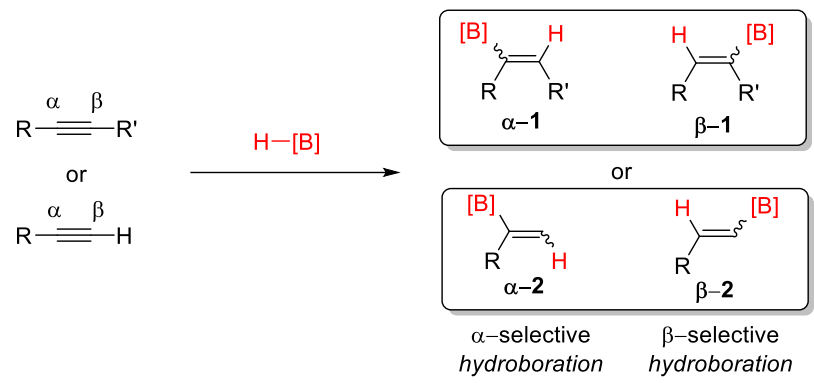

Scheme 2. Stereo- and regioselective hydroboration of internal and terminal alkynes.

This review summary is based on the availability of published reports of regio- and stereoselective catalytic hydroboration of alkynes, containing detailed descriptions of syn-hydroboration and antihydroboration. Although a few previously published reviews summarize the hydroboration of unsaturated compounds, none of them dealt with selective alkyne hydroboration to provide a synthetically useful compound alkenylboron compounds. ${ }^{[7-24]}$ Few review reports are also available which summarize the hydroelementation of olefins with specific catalysts $^{[7,14,16,18-20,22,24]}$ or specific substrates, ${ }^{[12]}$ however, the reviews are too limited to a certain subject and touch very small area of mono-selective hydroboration of alkynes. Based on our observations, over the past few decades, regio- and stereoselective overview of alkyne hydroboration is a much-discussed topic. We believe that it should be comprehensively reviewed to collate all the relevant research in this field. This review covers reports published prior to 2021 .

\section{2 syn-Hydroboration of alkynes}

\subsection{Transition metal-catalyzed hydroboration}

The main attention of catalytic hydroboration of alkynes is the opportunity to control the stereochemistry of the products it affords. Metal catalysts play a key role in selectivity. There are two main mechanisms used to achieve the synhydroboration of alkynes catalyzed by metal precursors, as shown in Scheme 3. Initially, an oxidative addition to the metal center occurs to afford an $\mathrm{M}-\mathrm{H}$ (as in 1) or $\mathrm{M}-[\mathrm{B}]$ (as in 4) bond.
Subsequently, this metal hydride or $\mathrm{M}-[\mathrm{B}]$ species is added to the alkyne moiety in a cis-manner, either via hydrometallation (path a) or boryl metallation (path b) to afford $\mathbf{2}$ or $\mathbf{5}$, respectively. Later, boryl demetallation or protonation of alkenyl metal species provides the stereospecific syn-hydroborated product (Scheme 3).
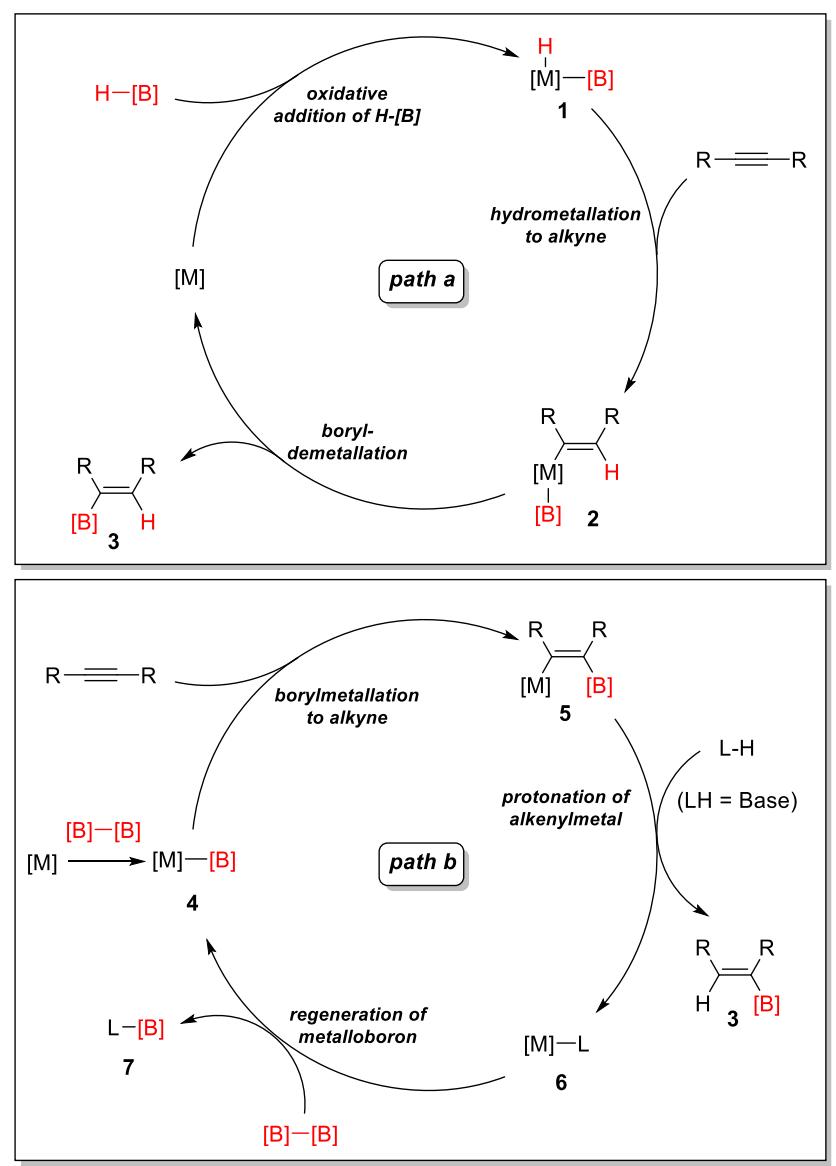

Scheme 3. Mechanistic aspect of syn-hydroboration of alkynes.

In a pioneering report on hydroboration of alkynes, Suzuki et al. described how conjugated enyne 8 undergoes selective hydroboration onto the alkyne moiety to afford allenic boronate 9 in the presence of $\mathrm{Pd}\left(\mathrm{PPh}_{3}\right)_{4}$ as a catalyst. Upon quenching 9 with benzaldehyde, homopropargylic alcohol $\mathbf{1 0}$ was produced (Scheme 4a). ${ }^{[29]}$ Using similar reaction conditions, a synthetic application of the Pd-catalyzed syn-hydroboration was reported for the synthesis of (E)-Doxepin. ${ }^{[30]}$ In 1996, Pereira and Srebnik introduced an Rh-catalytic system for the $\beta$-selective syn-hydroboration of terminal alkynes using benchtop stable HBpin (Scheme 4b). ${ }^{[31]}$ Their report revealed that a nickel catalyst $\left[\mathrm{NiCp}\left(\mathrm{PPh}_{3}\right) \mathrm{Cl}\right]$ can work as efficiently as an Rh catalyst to afford the $E$ alkenylborane compound. ${ }^{[31]}$ An application of the $\beta$ selective $s y n$-hydroboration of terminal alkynes was reported using an immobilized heterogeneous $\mathrm{Rh}$ catalytic system of $\mathrm{ClRh}\left[\mathrm{P}\left(\mathrm{CH}_{2} \mathrm{CH}_{2} \mathrm{R}_{6}\right)_{3}\right]_{3} \quad(\mathrm{R}=$ $\left.\left.\left(\mathrm{CF}_{2}\right)_{5} \mathrm{CF}_{3}\right)_{3}\right)$ in $\mathrm{CF}_{3} \mathrm{C}_{6} \mathrm{~F}_{11}$ solution. Significantly, the 
catalyst was reused for up to three catalytic cycles with a high turnover number (TON). ${ }^{[32]}$ Later, in 2004, $\beta$ selective syn-hydroboration of aromatic triyne was reported to afford the tris(boryl)vinyl product 14 (Scheme 4c). ${ }^{[33]}$ Subsequently, Suzuki cross-coupling of multi-alkenylborane compounds led to a variety of substrates containing chromophore units to yield fluorescent dye-substituted products. Given the importance, further studies on Rh-catalytic systems for stereoselective hydroboration of alkynes were carried out, ${ }^{[34,35]}$ showing that a rhodium hydride species could efficiently catalyze the selective hydroboration of 3,3,3-trifluoroprop-1-yne to produce $E$-selective product 15 (Scheme 4d). ${ }^{[35]}$

(a) Pd-catalyzed hydroboration of conjugated enyne (Suzuki, 1989)

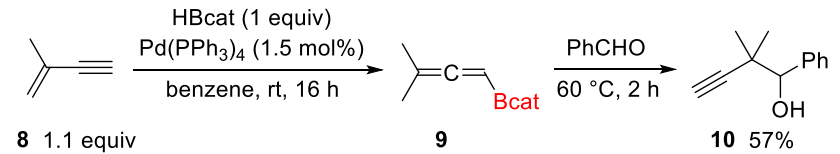

(b) Rh-catalyzed syn-hydroboration of terminal alkyne (Srebnik, 1996)

$$
{ }^{n} \mathrm{Hex}=\mathrm{H} \frac{\mathrm{Rh}(\mathrm{CO})\left(\mathrm{PPh}_{3}\right)_{2} \mathrm{Cl}(1 \mathrm{~mol} \%)}{\mathrm{DCM}, 25{ }^{\circ} \mathrm{C}, 3 \mathrm{~h}}{ }_{{ }^{n} \mathrm{Hex}}^{\substack{\mathrm{HB} \text {-selective } \\ 99 \%(\beta: \alpha=99: 1)}}
$$

(c) Rh-catalyzed stereo-selective hydroboration of aromatic triyne (Kand and Ko, 2004)

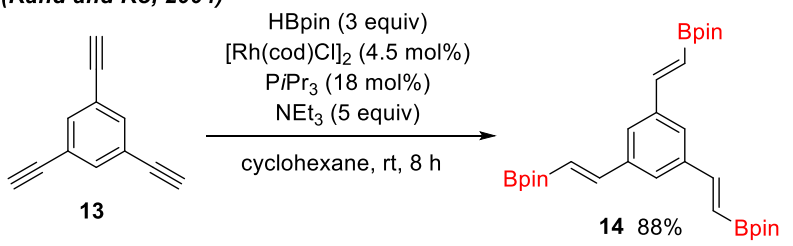

(d) Rh-catalyzed stereo-selective hydroboration of 3,3,3-trifluoropropyne (Braun, 2018)

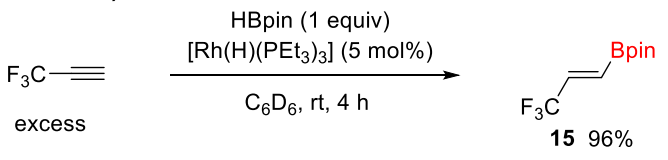

Scheme 4. (a) Early report of Pd-catalyzed stereoselective hydroboration. Rh-catalyzed hydroboration of (b) 1-octyne, (c) aromatic triyne, and (d) 3,3,3-trifluoropropyne.

Apart from the Rh-catalytic systems, various other noble metals, for instance, $\operatorname{Ir},{ }^{[36,37]} \mathrm{Ru},{ }^{[38,39]} \mathrm{Pt},{ }^{[40]}$ $\mathrm{Au},{ }^{[41]}$ and $\mathrm{Ag}^{[42-44]}$ proved their efficacy in the stereoselective hydroboration of alkynes. It is noteworthy that even a zirconocene hydride $\left(\mathrm{HZrCp} \mathrm{p}_{2} \mathrm{Cl}\right)$ efficiently catalyzed hydroboration of 1alkyne, in which $\beta$-selective syn-hydroboration proceeded almost exclusively, affording a trace of an anti-hydroboration product (Scheme 5a). ${ }^{[4]}$ Notably, in the case of internal alkynes, selectivity is retained. A simplified catalytic cycle proposed by the author suggests that hydrometallation followed by boryl demetallation is the key step in obtaining high stereoselectivity (Scheme 5b). ${ }^{[4]}$ Recently, a Zrcatalyzed system was established for the $\beta$-selective syn-hydroboration of terminal alkynes using diisopropylaminoborane (Scheme 5c). ${ }^{[46]}$ In this reaction, a catalytic amount of Grignard reagent is required to catalytically transform the $\mathrm{di}-i s o$ propylamineborane complex (DIPAB; $\left.\mathrm{H}_{3} \mathrm{~B} \leftarrow \mathrm{NH}(i \mathrm{Pr})_{2}\right)$ to di-iso-propylaminoborane (DIPOB; $\left.\mathrm{H}_{2} \mathrm{~B}-\mathrm{N}(i \mathrm{Pr})_{2}\right)$.

(a) Zr-catalyzed syn-hydroboration of terminal alkyne (Srebnik, 1995)

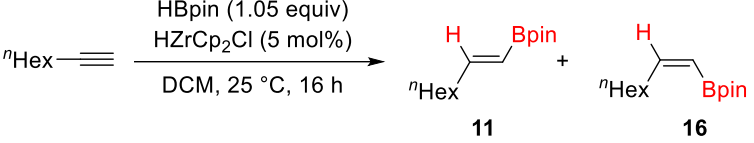

$$
\begin{aligned}
& \text { syn-hydroboration anti-hydroboration } \\
& \text { 93\% (syn:anti }=98: 2 \text { ) }
\end{aligned}
$$

(b) Mechanism of Zr-catalyzed syn-hydroboration (Srebnik, 1996)

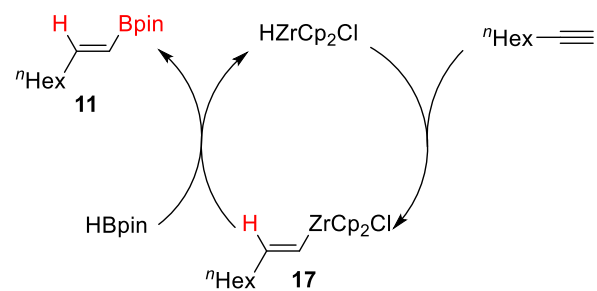

(c) Zr-catalyzed syn-hydroboration using diisopropylaminoborane (Pucheault, 2020)

$$
{ }^{n} \mathrm{Bu}=+\mathrm{H}_{3} \mathrm{~B} \leftarrow \mathrm{NHiPr}_{2} \underset{\begin{array}{c}
\text { ii) } \mathrm{HZrCp} \mathrm{Cl}_{2}(12 \mathrm{~mol} \%) \\
\text { methyl tert-butyl ether } \\
70^{\circ} \mathrm{C}, 16 \mathrm{~h}
\end{array}}{5 \mathrm{PIPAB}) \quad{ }_{\mathrm{Bu}}}
$$

Scheme 5. (a) Zr-catalyzed syn-hydroboration and (b) its mechanism. (c) Stereoselective hydroboration of terminal alkyne catalyzed by $\mathrm{Zr}$ using diisopropylaminoborane.

Given the importance of alkenylborane compounds in synthetic organic chemistry, various protocols have been developed to finetune the stabilization of alkenylborane synthons. A notable contribution was made by Suginome et al. to the Ir-catalyzed synhydroboration of alkynes using naphthalene-1,8diaminato $(\mathrm{HB}(\mathrm{dan}))$ (Scheme 6a). In the resultant product, the boronic acid moiety is masked by diaminonaphthalene, which can easily be demasked in the presence of $\mathrm{HCl}$ (aq.) to give reactive boric acid. Significantly, the masked alkenylboronic acid contained a $\mathrm{C}\left(\mathrm{sp}^{2}\right)-\mathrm{Br}$ bond (20) that easily experienced the Suzuki-Miyaura coupling reaction without disturbing the alkenylboron moiety (21, Scheme 6a). ${ }^{[36]}$ This report was a significant contribution. Recently, Yoshida and coworkers synthesized anthranilamide-substituted borane $[\mathrm{HB}$ (aam) $]$ as a stable and easy-to-handle substitute for $\mathrm{HB}$ (pin) for use in the Ir-catalyzed syn-selective hydroboration of terminal alkynes $(\mathbf{2 2}$, Scheme $6 \mathrm{~b}){ }^{[37]}$ 


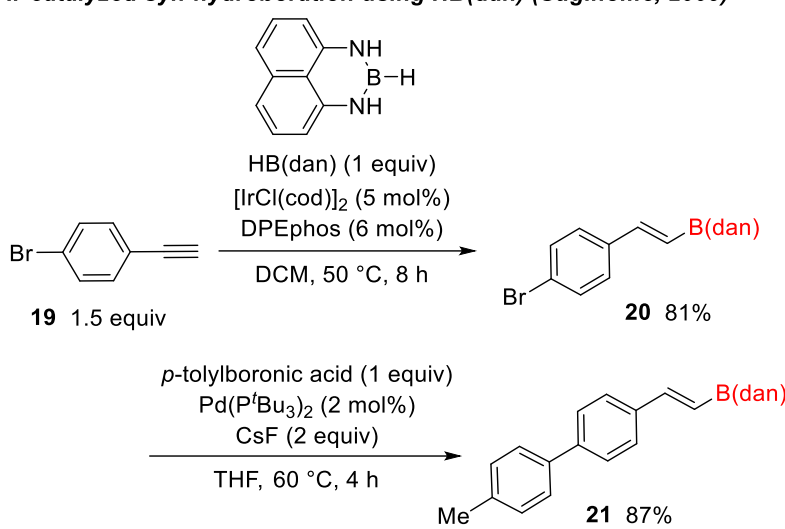

(b) Ir-catalyzed syn-hydroboration using HB(aam) (Yoshida, 2019)

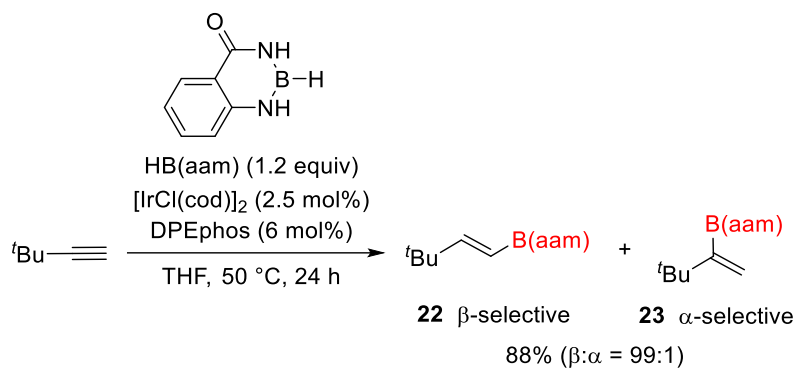

Scheme 6. Ir-catalyzed $\beta$-selective hydroboration of terminal alkynes using (a) $\mathrm{HB}$ (dan) and (b) $\mathrm{HB}$ (aam).

In 2009, Corma et al. reported the gold-catalyzed chemoselective hydroboration of alkynes. The authors showed that hydroboration occurred chiefly in terminal alkynes compared to terminal alkenes, yielding a substantial amount of $\mathbf{2 5}$ along with a minimal amount of $\mathbf{2 6}$ (Scheme 7a). They proposed that a gold-borane intermediate species was generated as an active species, rather than a gold-alkyne species. ${ }^{[41]}$ In 2014, Yoshida et al. showed that an Ag$N$-heterocyclic carbene complex has the qualities of a potent catalyst for the $\beta$-selective syn-hydroboration of terminal alkynes (Scheme 7b). ${ }^{[42]}$ It was hypothesized that the reaction was triggered by the formation of borylsilver(I) species. ${ }^{[47]}$ Subsequently, boryl metallation to alkynes proceeded largely in synfashion, followed by protonation by solvent $\mathrm{MeOH}$, resulting in the hydroboration product (11). Recently, in two parallel reports, both the Rit and Bi working groups showcased that silver salt $\left(\mathrm{AgSbF}_{6}\right.$ or $\left.\mathrm{AgOAc}\right)$ catalyzed the hydroboration of terminal alkynes using HBpin to exclusively yield syn-hydroborated $E$ alkenylborane 27 (Scheme 7c and 7d). ${ }^{[43,44]}$ Unlike the previously reported reaction catalyzed by the $\mathrm{Ag}-\mathrm{N}$ heterocyclic carbene complex, the authors hypothesized that a radical pathway was involved, which resulted in the formation of an exclusive $\beta$ selective product. Apart from homogeneous catalytic systems, heterogeneous catalysts have also proved to be potent catalysts for the syn-hydroboration of alkynes. For instance, $N$-heterocyclic thione ligandstabilized platinum nanoparticles displayed efficient reactivity in the hydroboration of terminal or internal alkynes with significant selectivity for the synhydroborated product. ${ }^{[40]}$ Hydroboration of terminal and internal alkynes using effective immobilization of $\mathrm{Ru}(\mathrm{CO}) \mathrm{Cl}(\mathrm{H})\left(\mathrm{PPh}_{3}\right)_{3}$ in ionic liquids or in biphasic ionic liquid/supercritical $\mathrm{CO}_{2}$ systems have also been reported, in which high syn-selectivity is maintained. ${ }^{[38,39]}$

(a) Au-catalyzed stereo- and regio-selective hydroboration (Corma, 2009)

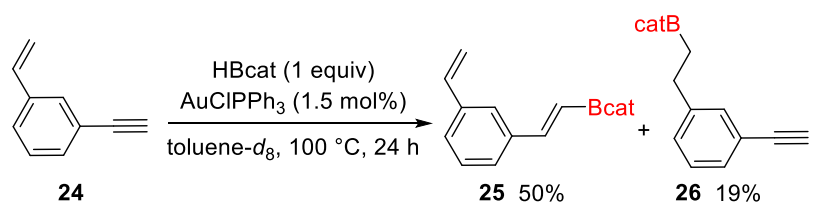

(b) Ag-catalyzed $\beta$-selective syn-hydroboration (Yoshida, 2014)

$$
\begin{aligned}
& { }^{n} \mathrm{Hex}=\frac{\begin{array}{c}
\text { pinB-Bpin (1 equiv) } \\
\text { (IMes)AgCl }(2 \mathrm{~mol} \%) \\
\mathrm{KOtBu}(6 \mathrm{~mol} \%)
\end{array}}{\mathrm{MeOH}, 50{ }^{\circ} \mathrm{C}, 2.5 \mathrm{~h}}{ }^{{ }^{n} \mathrm{Hex}}{ }^{\mathrm{Bpin}}+{ }^{n} \mathrm{Hex} \\
& 89 \%(\beta: \alpha=92: 8)
\end{aligned}
$$

(c) Ag-catalyzed syn-hydroboration of terminal alkyne (Rit, 2019)

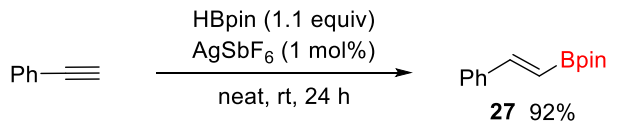

(d) Ag-catalyzed syn-hydroboration of terminal alkyne (Bi, 2019)

$$
\mathrm{Ph} \equiv \underset{\text { toluene, } 120^{\circ} \mathrm{C}, 18 \mathrm{~h}}{\stackrel{\operatorname{AgOAc}(10 \mathrm{~mol} \%)}{\longrightarrow}} \stackrel{\mathrm{Ph}}{\underset{27}{\mathrm{ABpin}}(1.5 \mathrm{equiv})}
$$

Scheme 7. (a) Au-catalyzed stereo- and position-selective hydroboration. (b-d) Ag-catalyzed stereoselective hydroboration of terminal alkynes.

$3 \mathrm{~d}$ transition metals are earth-abundant and demonstrate less toxic behavior, which makes them one of the prior choices for catalysis. In this regard, various first-row transition metals are also used as efficient catalysts in syn-selective hydroboration, for instance, $\mathrm{Ti},{ }^{[48,49]} \mathrm{Mn},{ }^{[50]} \mathrm{Fe},{ }^{[51-58]} \mathrm{Co},{ }^{[59-61]} \mathrm{Ni},{ }^{[62]}$ $\mathrm{Cu},{ }^{[63-77]}$ and $\mathrm{Zn} .{ }^{[78]}$

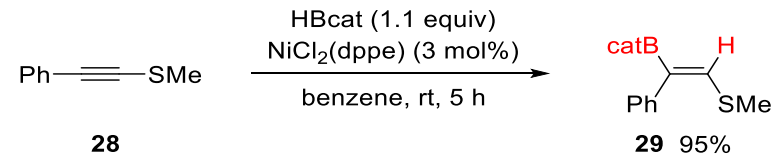

Scheme 8. Ni-catalyzed regio- and stereoselective hydroboration of thioacetylene (Miyaura and Suzuki, 1993).

One of the early reports of regio- and stereoselective hydroboration of 1-(alkylthio)-1-alkyne (28) using $\mathrm{HBcat}$ and $\mathrm{NiCl}_{2}$ (dppe) as catalysts (Scheme 8) was published by Miyaura and Suzuki in 1993. ${ }^{[62]}$ Notably, hydroboration proceeded exclusively on the $\beta$-position with respect to the thioalkyl group. Unfortunately, this catalytic system did not show significant efficacy in 
terms of selectivity when only an aliphatic or aromatic substituted alkyne (1-octyne or 1-phenyl-1-propyne) was used as the substrate. ${ }^{[62]}$ Soon after, Hartwig et al. showcased a competent catalytic protocol for the synhydroboration of alkynes with HBcat using readily available dicarbonyltitanocene as a catalyst (Scheme 9 a). In a plausible mechanism, it was shown that $\sigma$ bond metathesis proceeded with $\mathrm{HBcat}$ and metallacyclopropene generated from the reaction between alkynes and the titanocene complex. ${ }^{[48]}$ Very recently, Panda et al. synthesized a titanium(IV) complex supported by dianionic amidophosphine-borane ligands, which displayed high efficacy toward the $\beta$-selective syn-hydroboration of terminal alkynes (Scheme 9b). ${ }^{[49]}$ Geetharani and coworkers also reported that a rare-earth metal of group $3(\mathrm{Sc})$ works as an efficient catalyst in the synselective hydroboration of alkynes (Scheme 9c). ${ }^{\text {[79] }}$ Commercially available Lewis acid $\mathrm{Sc}(\mathrm{OTf})_{3}$ was used as a catalyst along with a catalytic amount of $\mathrm{NaBHEt}_{3}$, which successfully syn-hydroborated a series of the terminal as well as internal alkynes. A general mechanism for $s y n$-selective hydroboration of alkyne using Ti or Sc as the catalyst is postulated in Scheme $9 \mathrm{~d}$ which is in principle different from the general mechanism presented in Scheme 3. At first, a metalhydride $\mathbf{3 1 A}$ is thought to be produced which added to alkyne in a syn-fashion via 31B. In the last step, metalalkene species $31 \mathrm{C}$ reacted with another molecule of hydroboron compound to produce the synhydroborated product along with the regeneration of metal-hydride species. ${ }^{[49,79]}$ (a) Ti-catalyzed selective hydroboration of alkyne (Hartwig, 1996)

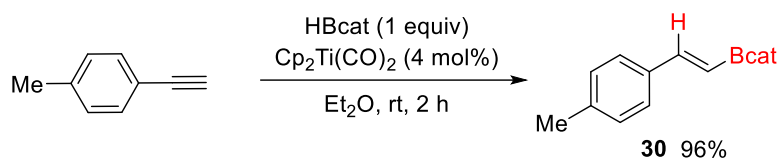

(b) Ti-catalyzed selective hydroboration of alkyne (Panda, 2020)

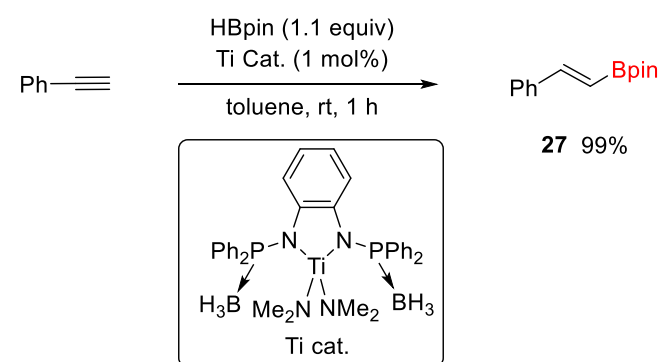

(c) Lewis acidic $\mathrm{Sc}(\mathrm{OTf})_{3}$-catalyzed selective hydroboration of alkyne (Geetharani, 2018)

$$
\mathrm{Ph} \equiv \quad \underset{\begin{array}{l}
\text { HBpin (1.2 equiv) } \\
\mathrm{Sc}(\mathrm{OTf})_{3}(5 \mathrm{~mol} \%)
\end{array}}{\stackrel{\mathrm{NaBHEt}_{3}(5 \mathrm{~mol} \%)}{\longrightarrow}} \quad
$$

(d) General mechanism for Ti or Sc catalyzed hydroboration of alkyne

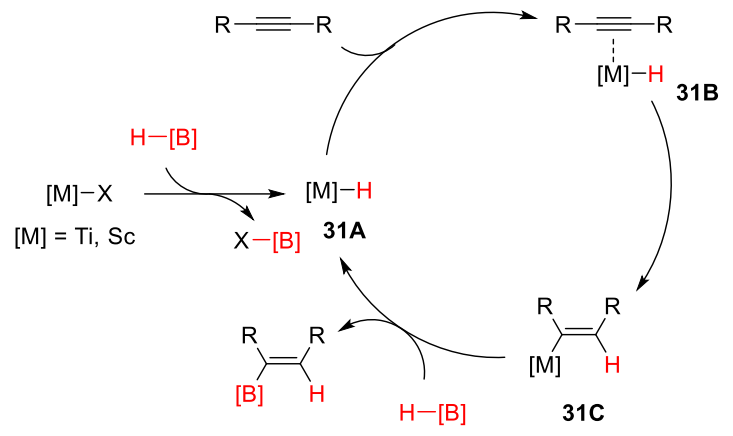

Scheme 9. (a) and (b) Ti-catalyzed syn-selective hydroboration of alkynes. (c) $\mathrm{Sc}(\mathrm{OTf})_{3}$-catalyzed synselective hydroboration. (d) General mechanism for synhydroboration of alkyne using Ti or Sc as catalysts.

$\mathrm{Cu}$ catalysts have contributed significantly to hydroboration of alkynes in a highly stereo- and regioselective manner. ${ }^{[63-77]}$ It is worthy of mention that the $\beta$ - or $\alpha$-selective borylation of aromatic substituted unsymmetrical internal alkynes (such as $\mathbf{3 2}$ or 34) was achieved by varying the borylating reagents. The Yun, Tsuji, and Cazin groups independently reported a similar $\mathrm{Cu}$-catalytic system using $\mathrm{B}_{2} \operatorname{pin}_{2}$ as the borylating precursor to achieving $\beta$-selective synhydroboration products $33 \boldsymbol{\beta}$ or $\mathbf{3 5 \beta}$ (Scheme 10). ${ }^{[63,64,74]}$ Likewise a Cu-photocatalytic system was also developed by Poisson et al for obtaining $\beta$ selective syn-hydroboration of internal alkynes with complete selectivity (Scheme 10d) ${ }^{[69]}$ Conversely, $\alpha$ selective syn-hydroboration product $\mathbf{3 5 \alpha}$ was obtained when HBpin was used in a Cu-catalytic system mentioned in Scheme 11. ${ }^{[64,74,77]}$ 
(a) Cu-catalyzed $\beta$-selective syn-hydroboration (Yun, 2011)

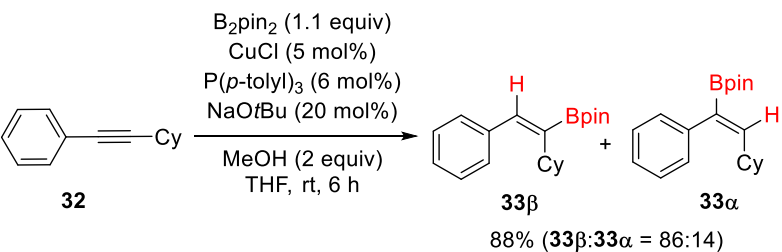

(b) Cu-catalyzed $\beta$-selective syn-hydroboration (Tsuji, 2012)

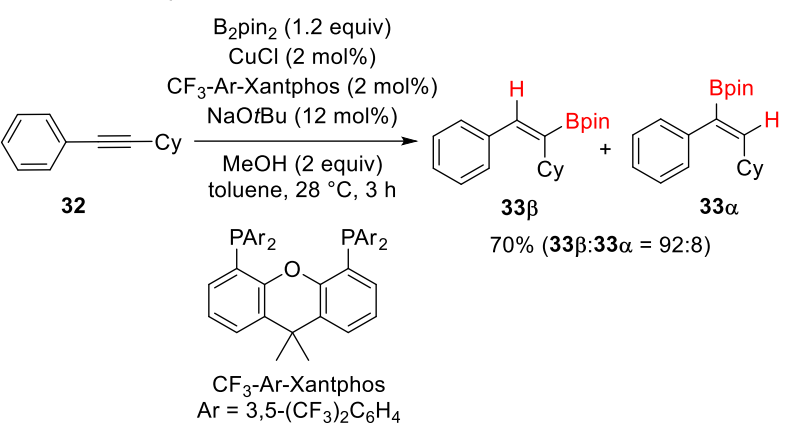

(c) Cu-catalyzed $\beta$-selective syn-hydroboration (Cazin, 2014)

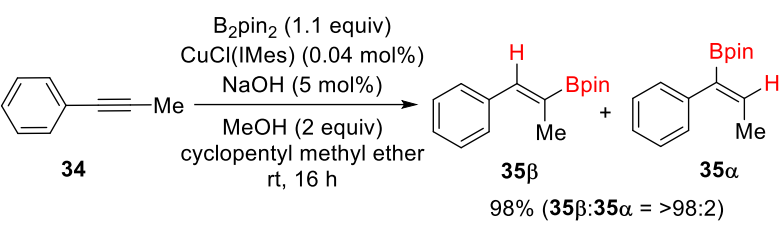

(d) Cu-photocatalyzed $\beta$-selective syn-hydroboration (Poisson, 2021)

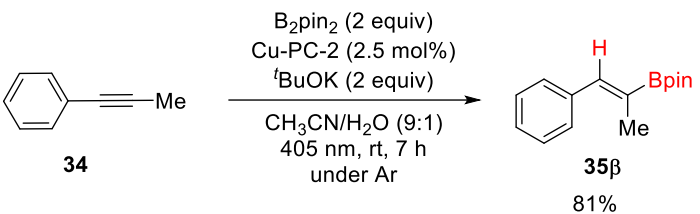

Scheme 10. (a)-(c) Cu-catalyzed $\beta$-selective synhydroboration of internal alkynes. (d) Cu-photocatalyzed $\beta$ selective syn-hydroboration of internal alkynes. (a) Cu-catalyzed $\alpha$-selective syn-hydroboration (Tsuji, 2012)

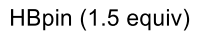

$\mathrm{CH}_{3}$-Ar-Xantphos (2 mol\%)
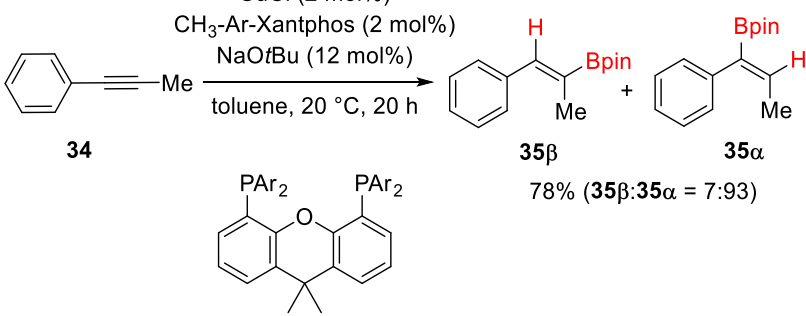

$\mathrm{CH}_{3}$-Ar-Xantphos $\mathrm{Ar}=3,5-\left(\mathrm{CH}_{3}\right)_{2} \mathrm{C}_{6} \mathrm{H}_{4}$

(b) Cu-catalyzed $\alpha$-selective syn-hydroboration (Cazin, 2014)

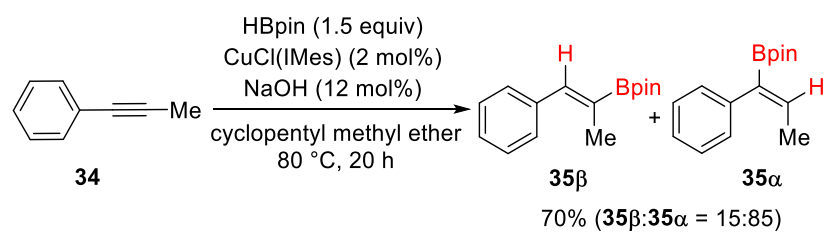

(c) Cu-catalyzed $\alpha$-selective syn-hydroboration (Whittlesey, 2018)

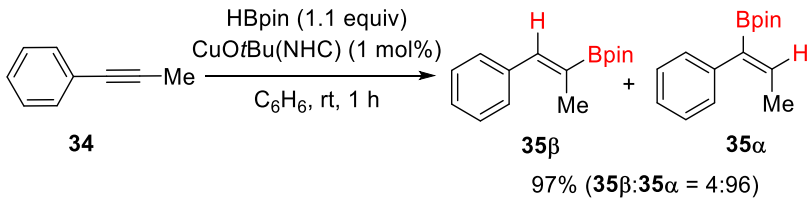

Scheme 11. (a)-(c) Cu-catalyzed $\alpha$-selective synhydroboration of internal alkynes.

Complementary $\beta$ - and $\alpha$-selective syn-hydroboration proceed based on the use of borylating reagent. For clarity, two plausible catalytic cycles are presented in Scheme 12. A boryl copper species (36) is responsible for the $\beta$-selective product $\mathbf{3 8}$ (path a, Scheme 12), while a copper hydride species (39) was formed from the reaction between $\mathrm{Cu}$-salt and $\mathrm{HBpin}$, which is in charge of the formation of $\alpha$-selective product 41 (path b, Scheme 12). Following the formation of copper hydride 39, hydrocupration to alkyne proceeded to yield 40, which showed high regioselectivity owing to the directing effect of phenyl substitution. Later boryl demetallation afforded a high $\alpha$-selective syn-hydroborated product (41) (path b, Scheme 12). ${ }^{[64]}$ 

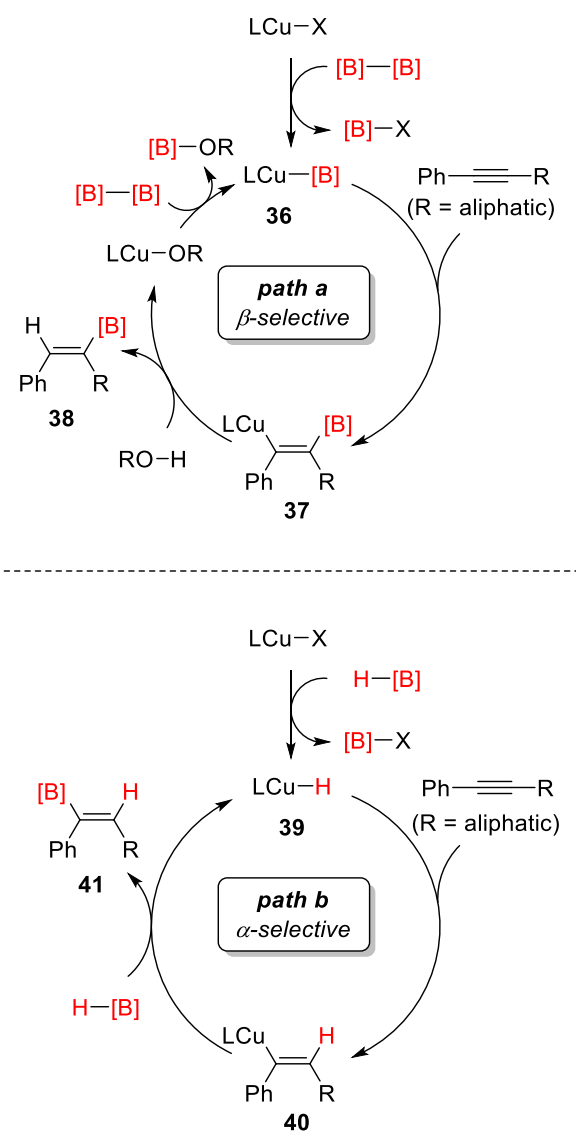

Scheme 12. Mechanistic differences in $\beta$ - and $\alpha$-selective borylation of unsymmetrical aromatic substituted alkynes, using $\mathrm{B}_{2} \mathrm{pin}_{2}$ and HBpin, respectively.

Substituted functional groups on alkynes have also contributed notably in controlling $\alpha$ - or $\beta$-selective product formation in a $\mathrm{Cu}$-catalytic system. In this regard, the Arrayás and Carretero group reported that the presence of a propargylic polar group, such as $\mathrm{OH}$, $\mathrm{OR}, \mathrm{SAr}, \mathrm{SO}_{2} \mathrm{Ar}$, and NHTs allowed excellent $\beta$ selectivity ( $\beta$ to the propargylic function, as shown in 42, Scheme 13). ${ }^{[70]}$ McQuade et al. reported that regioselective syn-hydroboration of propargylic alcohols and ethers can also occur, providing either an $\alpha$ - or $\beta$-selective product depending on the starting materials - propargylic alcohols $(\mathbf{4 3})$ or ethers $(47)$ and NHC ligand on the copper complex. ${ }^{[71]}$ Chalcogensubstituted alkynes also control the regioselectivity of a syn-hydroboration reaction (as shown in 44-46 and 48). ${ }^{[67,68,73]}$ Given the importance of selective hydroboration reactions and considering the high efficacy of the copper catalytic system, a few heterogeneous $\mathrm{Cu}$-catalytic systems have been reported in the hydroboration of alkynes with high synselectivity. ${ }^{[66,72,76]}$

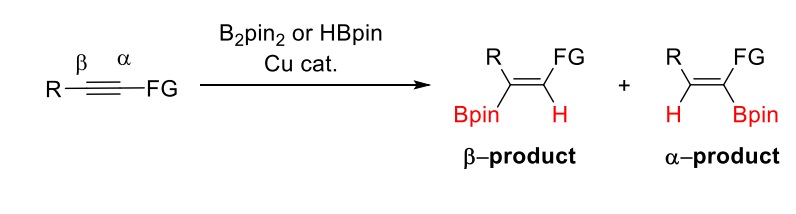

$\beta$-selective syn-hydroboration
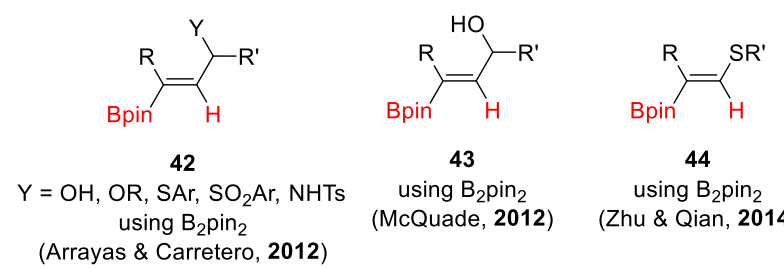

using $\mathrm{B}_{2}$ pin $_{2}$

(Zhu \& Qian, 2014)

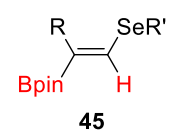

using $\mathrm{B}_{2}$ pin $_{2}$ (Moro, 2020)

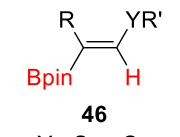

$\mathrm{Y}=\mathrm{S}$ or $\mathrm{Se}$

using $\mathrm{B}_{2}$ pin $_{2}$

(Oliveira \& Stefani, 2020)

$\alpha$-selective syn-hydroboration

$$
\begin{gathered}
47 \\
\mathrm{Ar}=4-\mathrm{NO}_{2} \mathrm{C}_{6} \mathrm{H}_{4} \\
\text { using } \mathrm{B}_{2} \mathrm{pin}_{2} \\
\text { (McQuade, 2012) }
\end{gathered}
$$

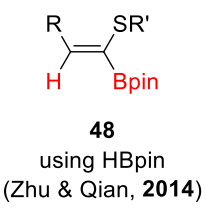

Scheme 13. Various Cu-catalyzed functional groups directed $\alpha$ - or $\beta$ - selective syn-hydroboration.

Bearing in mind the importance of earth-abundant metal catalysts in selective hydroboration reactions, Fe-based complexes have been widely studied. Notably, in 2013, Enthaler et al. reported the first example of a $\mathrm{Fe}_{2}(\mathrm{CO})_{9}$ catalytic system for the selective hydroboration of alkynes (Scheme 14a). ${ }^{[52]}$ This protocol selectively provided syn-hydroborated $E$-alkene as a product when terminal alkynes were used as the starting material. However, in the case of an unsymmetrical alkyne, a mixture of $\alpha$ - and $\beta$ borylated products were obtained. In the same year, Thomas et al. reported a bench-stable iron complex in combination with a bis(imino)pyridine ligand, which upon activation with Grignard reagent, proved to be an efficient system for the syn-selective hydroboration of alkynes (Scheme 14b). ${ }^{[51]}$ Later, Webster et al. introduced an alkylated iron complex (50) for the hydroboration of alkynes (Scheme 14c). ${ }^{[55]}$ However, the report shows a narrow substrate scope for unsymmetrically substituted internal alkynes, as a mixture of products was obtained. An iron-hydride complex (51) supported by a pincer ligand was employed by Nishibayashi and coworkers for the synhydroboration of internal alkynes to afford $E$-alkene (Scheme 14d) ${ }^{[56]}$ Recently, Findlater et al. presented a bis(2,6-diisopropylaniline)acenaphthene-ligated iron complex (52) for the hydroboration of terminal and internal alkynes (Scheme 14e). ${ }^{[57]}$ Around the same 
time, an iron-hydride complex (53) was used for the syn-hydroboration of unsymmetrical internal alkynes, which provided complementary regioselectivity based on the use of a boron source $\left(\mathrm{B}_{2} \mathrm{pin}_{2}\right.$ or HBpin; Scheme 14f). ${ }^{[58]}$ Furthermore, stereoselective hydroboration of alkynes was achieved using iron nanoparticles or ironbased heterogeneous catalysts. ${ }^{[53,54]}$

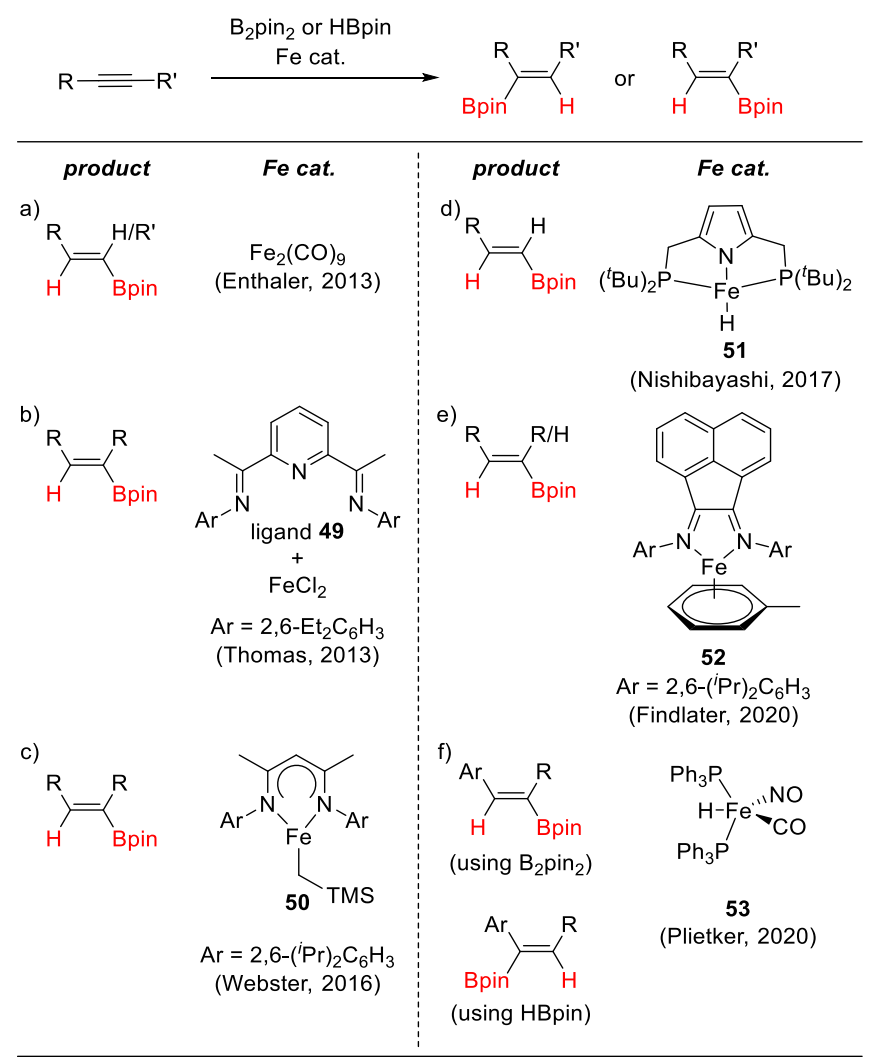

Scheme 14. (a)-(f) Various Fe-catalytic systems in synselective hydroboration.

A cobalt-catalytic system was introduced by Trovitch et al. for $s y n$-selective hydroboration using an $\alpha$-diimine cobalt hydride complex (55) as the catalyst. This protocol provides a decent substrate scope for terminal alkynes, affording $E$-alkenylboranes with high selectivity (Scheme 15a). ${ }^{[59]}$ Later, in 2019, Zhang et al. introduced a bench-stable and inexpensive coordination polymeric cobalt complex (58) as a highly efficient catalyst in combination with $\mathrm{KO}^{t} \mathrm{Bu}$ for the stereoselective syn-hydroboration of the terminal as well as internal alkynes (Scheme 15b). ${ }^{[61]}$ Notably, this catalytic system works efficiently even with very low catalyst loading and can provide a turnover frequency (TOF) of up to $47520\left(\mathrm{~h}^{-1}\right)$. Ge et al. reported a $\mathrm{Co}(\mathrm{acac})_{2}$-catalyzed regio-divergent stereoselective syn-hydroboration of symmetrical or unsymmetrical 1,3-diynes in the presence of a bisphosphine ligand (dppf or xantphos). Remarkably, excellent $\alpha$-selectivity was obtained when dppf was used as a ligand along with the cobalt pre-catalyst. However, opposite selectivity ( $\beta$-selective) was witnessed when xantphos was used as a ligand
(Scheme 15c). ${ }^{[60]} \mathrm{A}$ detailed experimental investigation suggested that $\operatorname{Co}(\text { acac })_{2} / \mathrm{dppf}$-catalyzed hydroboration proceeds through a cobalt boryl intermediate to afford $\mathbf{6 1 \alpha}$, while the hydroboration reaction catalyzed by $\mathrm{Co}(\mathrm{acac})_{2} / \mathrm{xantphos}$ proceeds via a cobalt-hydride intermediate to provide $61 \beta$ in significant quantity.

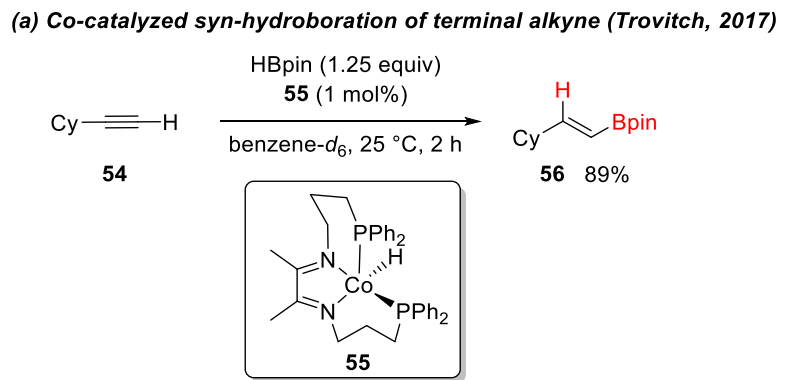

(b) Highly efficient Co-catalyzed syn-hydroboration (Zhang, 2019)

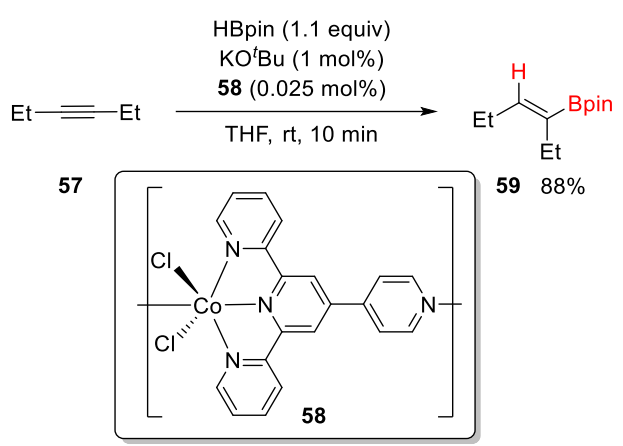

(c) Co-catalyzed regio-divergent syn-hydroboration of 1,3-diyne (Ge, 2019)

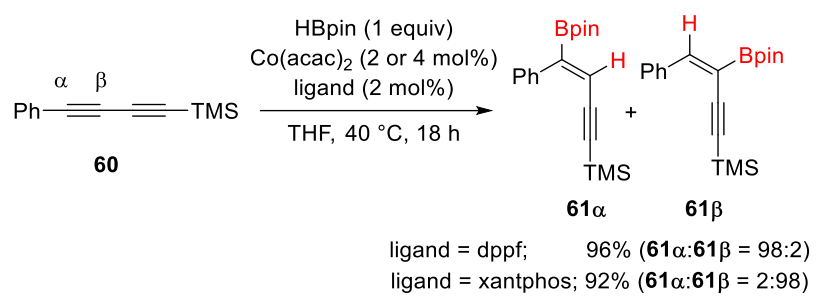

Scheme 15. Co-catalyzed syn-selective hydroboration of alkynes.

Syn-selective hydroboration of alkynes was reported using commercially available $\mathrm{Zn}(\mathrm{OTf})_{2}$ as a catalyst in combination with $\mathrm{NaBHEt}_{3}$ (Scheme 16a). ${ }^{[78]}$ A series of internal and terminal alkynes served as a substrate to yield the corresponding products in good quantity. Rueping et al. reported the hydroboration of propargylic alcohol (62) catalyzed by a manganese complex (63) to afford an $\alpha$-selective $s y n$-hydroboration product (Scheme 16b). ${ }^{[50]}$ Notably, along with propargylic alcohol, propargylic amines, and symmetrical internal alkynes participated efficiently in this Mn-catalyzed syn-hydroboration reaction to afford corresponding borylated alkenes. It was noted that the success of the reaction relies on the use of $\mathrm{NaBHEt}_{3}$, as it helped to form the catalytically active Mn species. 
(a) Zn-catalyzed syn-selective hydroboration of alkyne (Geetharani, 2019)

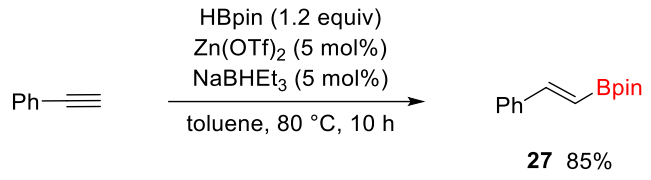

(b) Mn-catalyzed $\alpha$-selective syn-hydroboration of propargylic alcohols (Rueping, 2020).

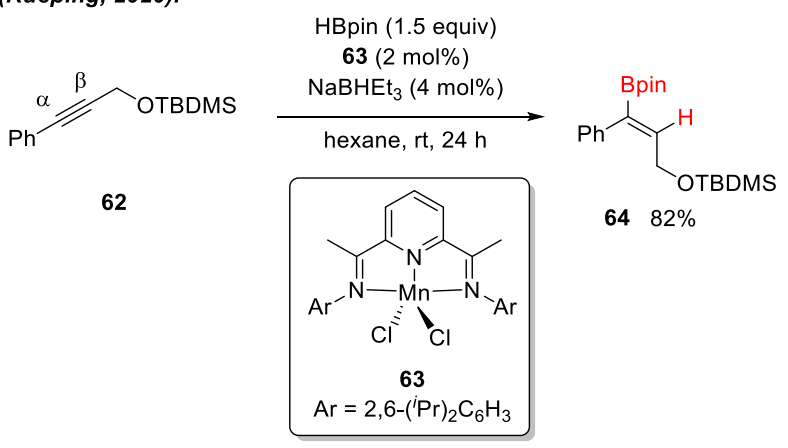

Scheme 16. (a) Zn- and (b) Mn-catalyzed synhydroboration of propargylic alcohols.

\subsection{Alkali and alkaline earth metal-catalyzed syn-hydroboration}

Having great worth of alkenyl organoboron compounds as synthetic intermediates, various catalysts have been designed for the selective hydroboration of alkynes. One of the finest contributions to this field was made when alkali and alkaline earth metals began to be used as catalysts. There are several advantages of using alkali and alkaline earth metal catalysts over transition metal catalysts, such as abundance, cost effectiveness, and easily separable techniques. The first selective hydroboration of alkynes, using a catalytic amount of $\mathrm{NaOH}$ powder as an initiator, was reported in 2017 (Scheme 17a). ${ }^{[80]}$ Several terminal alkynes were selectively hydroborated to form $E$-alkenes using this protocol. The initial mechanistic exploration suggested that an anionic boron hydride species, generated in-situ in the reaction mixture induced by $\mathrm{NaOH}$, facilitated the reaction. However, this hypothesis has not been proven yet. Another report appeared just a year later, which dealt with $\mathrm{NaOMe}$ as an initiator in the $s y n$-hydroboration of terminal as well as internal alkynes (Scheme 17b). ${ }^{[81]}$ Notably, a trace of anti-hydroborated product was also obtained during this process. (a) $\mathrm{NaOH}$-catalyzed syn-hydroboration of alkyne (Wu, Liu and Zhao, 2017)

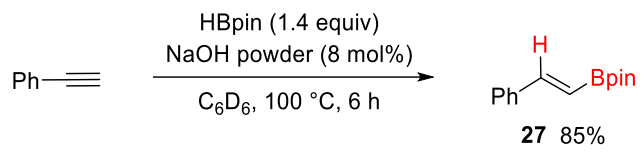

(b) NaOMe-induced syn-hydroboration of alkyne (Wen, 2018)

$$
\mathrm{Ph} \equiv \frac{\substack{\text { pinB-Bpin (1 equiv) } \\ \mathrm{NaOMe}(50 \mathrm{~mol} \%)}}{\mathrm{MeOH}, 40{ }^{\circ} \mathrm{C}, 10 \mathrm{~h}}
$$

Scheme 17. (a) and (b) Sodium salt as catalysts in the synselective hydroboration of alkynes.

(a) ${ }^{n}$ BuLi-catalyzed syn-hydroboration of alkyne (Bao and Xue, 2019)

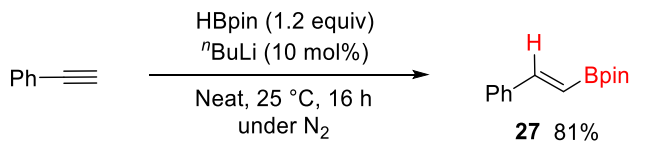

(b) ${ }^{n}$ BuLi-catalyzed syn-hydroboration of alkyne (Shi and Xu, 2019)

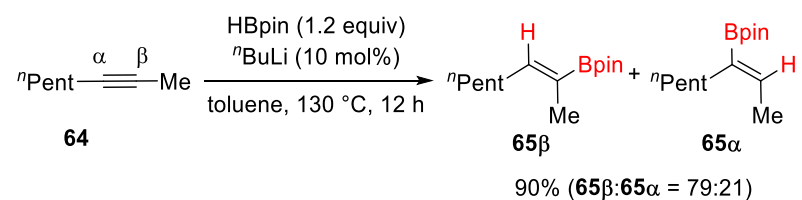

(c) Li compound-catalyzed syn-hydroboration of alkyne (Sen, 2019)

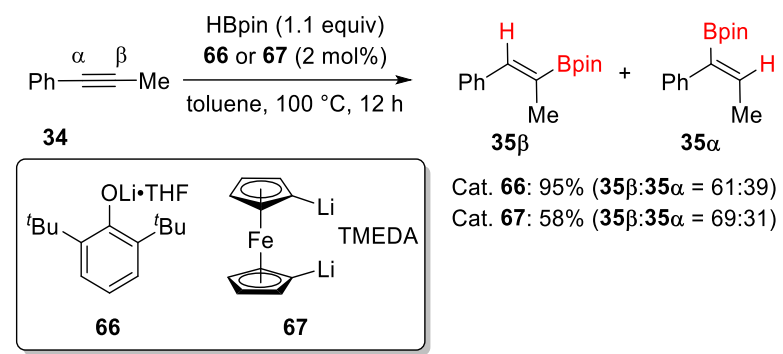

Scheme 18. (a)-(c) Lithium compounds as catalysts in the $s y$-selective hydroboration of alkynes.

It was found that even organolithium compounds can catalyze the hydroboration of alkynes. The $\mathrm{Bao} / \mathrm{Xue}$ and $\mathrm{Shi} / \mathrm{Xu}$ groups independently reported that $n$-butyl lithium can efficiently catalyze the hydroboration of terminal (Scheme $18 \mathrm{a})^{[82]}$ as well as internal alkynes (Scheme 18b). ${ }^{[83]}$ In the case of unsymmetrical internal alkynes, a mixture of products was obtained, with a major amount comprising $\mathbf{6 5 \beta}$. The computational study suggested that a zwitterionic intermediate, generated from the reaction of HBpin and $n \mathrm{BuLi}$, plays a key role in hydroboration. ${ }^{[82]}$ Soon after, Sen et al. introduced another efficient catalytic system in which a Li compound (66 or 67) catalyzed terminal or internal alkynes during hydroboration (Scheme 18c). In the case of unsymmetrical 
substituted internal alkynes, a mixture of products was obtained, with a higher portion constituted by the $\beta$ product (35及). ${ }^{[84]}$
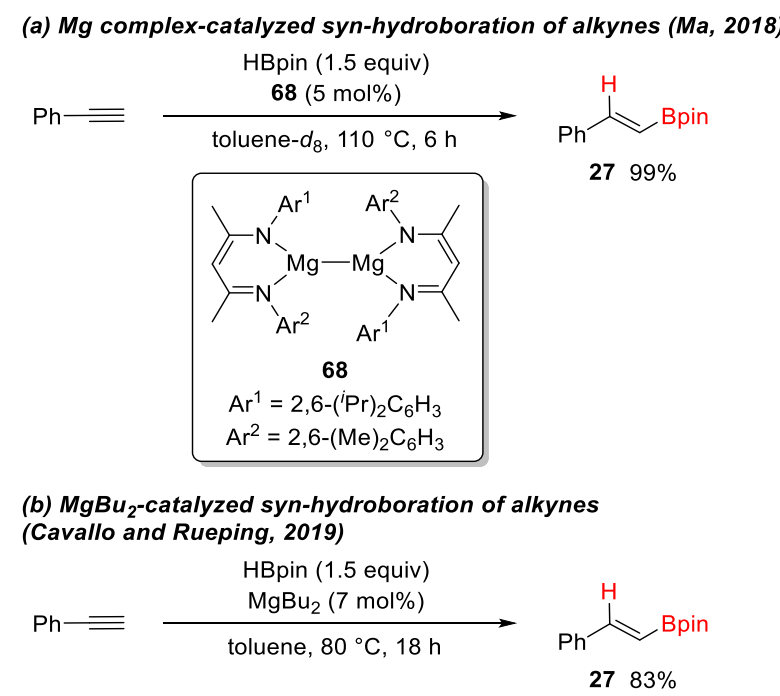

Scheme 19. (a) and (b) Mg-catalyzed syn-selective hydroboration of alkynes.

Ma et al. reported a di-magnesium complex (68) bearing an unsymmetrical $\beta$-diketimine ligand, which works as a reactive pre-catalyst in the syn-selective hydroboration of alkynes (Scheme 19a). ${ }^{[85]}$ The authors discovered the involvement of a magnesium hydride species as a reactive intermediate based on experimental and computational evidence. Soon after, Cavallo, Rueping, and coworkers showed that readily available $\mathrm{MgBu}_{2}$ can catalyze the same transformation with excellent syn-selectivity (Scheme 19b).$^{\left[{ }^{[6]}\right.}$ Along with terminal alkynes, various internal alkynes were also suitable candidates for this syn-hydroboration protocol.

\subsection{Aluminum-catalyzed syn-hydroboration}

As discussed in the first section, transition metal catalysts have contributed significantly to the selective hydroboration of alkynes. However, interest in sustainable chemical processes is growing and researchers are seeking replacements for transition metal catalysts. In this regard, the main group elements are comparable substitutes for transition metal catalysts. Aluminum-related compounds have contributed notably to the selective hydroboration of alkynes. In 2016, Roesky et al. first reported that an aluminum dihydride complex (69), bearing a $\beta$ diketiminate ligand, successfully catalyzed the synhydroboration of terminal alkynes (Scheme 20). ${ }^{[87]}$ Various aromatic groups-substituted as well as aliphatic group-substituted terminal alkynes reacted smoothly to afford the $E$-alkene products.

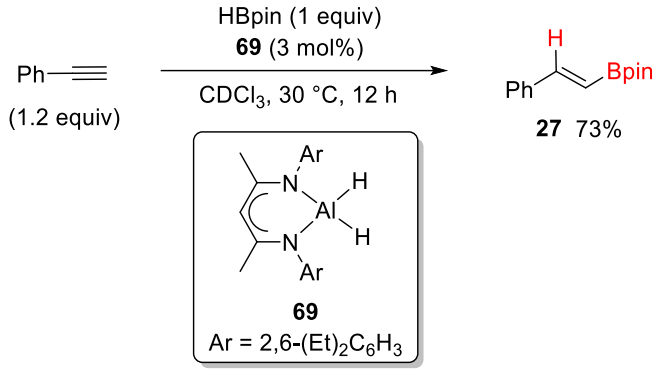

Scheme 20. Aluminum dihydride-catalyzed synhydroboration of terminal alkynes (Yang, Parameswaran, and Roesky, 2016).

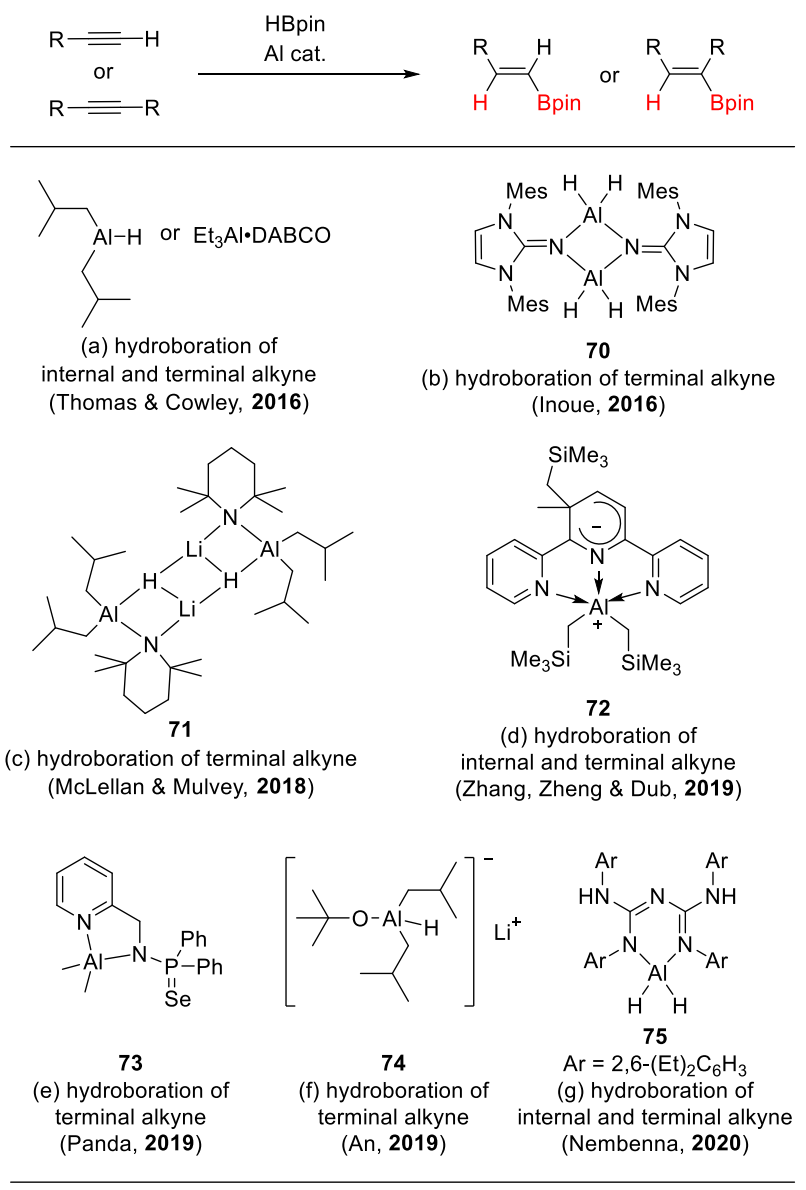

Scheme 21. (a)-(g) Various aluminum complexes work efficiently in the syn-selective hydroboration of internal and terminal alkynes.

Following the pioneering report by Roesky et al., ${ }^{[87]}$ various other aluminum complexes were explored for stereoselective hydroboration. For instance, the Thomas and Cowley groups reported that commercially available aluminum hydride DIBAL-H or bench-stable $\mathrm{Et}_{3} \mathrm{Al} \cdot \mathrm{DABCO}$ efficiently catalyzes the $s y n$-hydroboration of internal and terminal alkynes (Scheme 21a): ${ }^{[88]}$ Inoue et al. reported an aluminum hydride (70) stabilized by $N$-heterocyclic imines as a catalyst in the hydroboration of terminal alkynes (Scheme 21b); ${ }^{[89]}$ the McLellan and Mulvey groups 
reported a neutral aluminum lithium complex (71), which functioned cooperatively as a catalyst in the hydroboration of terminal alkynes (Scheme $21 \mathrm{c}$ ); ${ }^{[00]}$ a zwitterionic aluminum complex (72) was introduced by Zhang, Zheng, and Dub in the efficient synhydroboration of internal or terminal alkynes with a high TON (up to 1000) (Scheme 21d); ${ }^{[91]}$ Panda et al. introduced an aluminum complex (73) supported by a functionalized amidophosphine ligand for smooth hydroboration of terminal alkynes (Scheme 21e); ${ }^{[92]}$ lithium di-iso-butyl-tert-butoxyaluminum hydride (LDBBA; 74) was used by An et al. in the stereoselective hydroboration of terminal alkynes (Scheme 21f); ${ }^{[93]}$ and very recently, Nembenna et al. reported that conjugated bis-guanidinate (CBG)supported aluminum dihydride (75) proficiently catalyzed the syn-hydroboration of internal and terminal alkynes (Scheme 21g). ${ }^{[94]}$ Since various aluminum catalytic systems have been developed for alkyne syn-hydroboration, it is now easier to search for the right choice of Al-catalyst for a certain substrate. Furthermore, progress in this field is ongoing. A notable example of aluminum complex catalysis was reported by Hong, $\mathrm{Xu}$, and Shi in the regio- and stereoselective hydroboration of alkynylsilanes (Scheme 22). ${ }^{[95]}$ In particular, they found that several alkynylsilanes, bearing various functional groups and heterocyclic substituents, formed hydroborated products smoothly in good yields and with high selectivity.

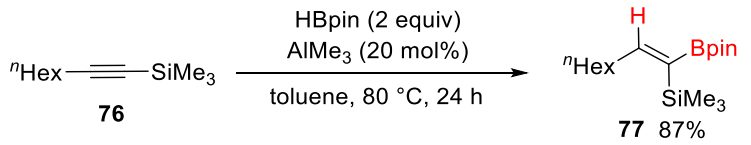

Scheme 22. Aluminum-catalyzed syn-hydroboration of alkynylsilanes (Hong/Xu/Shi, 2019).

Considering the stereoselective accuracy of aluminum-catalyzed hydroboration of internal and terminal alkynes, establishing the general mechanistic aspect of such hydroboration is important (Scheme 23). In an aluminum complex-catalyzed hydroboration reaction, the aluminum hydride species, which mainly catalyzes the reaction, is either used or generated insitu. In the case of a terminal alkyne, at first, deprotonation of the alkyne with aluminum hydride occurs, leading to the formation of aluminum acetylide (78) and a molecule of $\mathrm{H}_{2}$. Usually, this step is exothermic. The polarization of the $\mathrm{C} \equiv \mathrm{C}$ bond in $\mathbf{7 8}$ resulted in a cycloaddition of HBpin bond to the $\mathrm{C} \equiv$ $\mathrm{C}$ bond in a syn-fashion via a four-membered transition state to form 79. Again, a highly polarized bond of $\mathrm{Al}-\mathrm{C}$ in $\mathbf{7 9}$ favors the $\sigma$-bond metathesis with another molecule of alkyne to yield the synhydroborated product 80 (path a, Scheme 23). ${ }^{[87]}$ Conversely, in the case of internal alkynes, a different mechanism operates, in which the $\mathrm{Al}-\mathrm{H}$ bond of aluminum hydride is inserted into the alkyne triple bond in a syn-fashion to produce an intermediate $\mathbf{8 1}$.
Subsequently, in a reaction with HBpin, transmetallation occurs to form the desired synhydroborated product $\mathbf{8 0}$ along with the regeneration of aluminum hydride (path $b$, Scheme 23). ${ }^{[87,91,93,94]}$
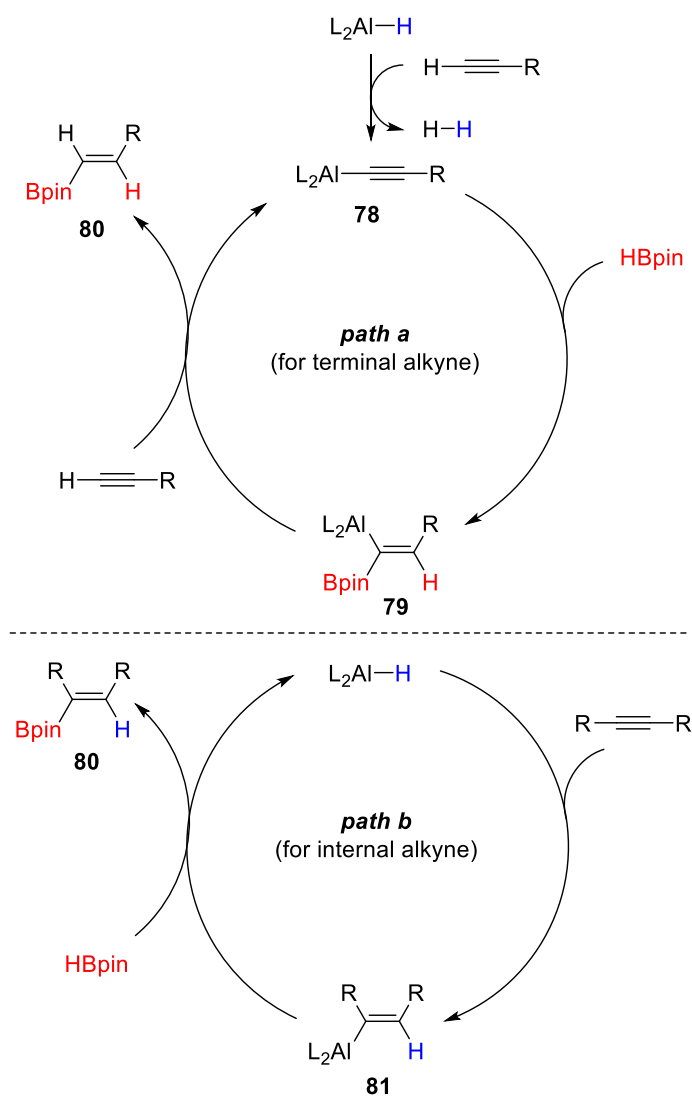

Scheme 23. Mechanistic aspects of aluminum-catalyzed hydroboration of internal and terminal alkynes.

\subsection{Metal-free catalytic syn-hydroboration}

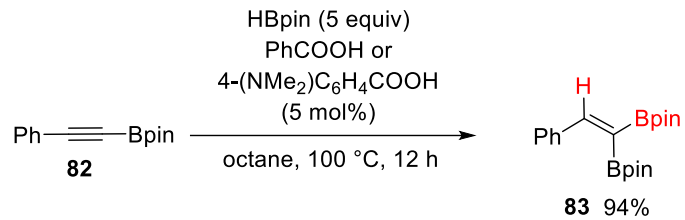

Scheme 24. A carboxylic acid-catalyzed syn-hydroboration of alkynes (Jin, 2014).

To eliminate the possibility of the incorporation of metals into a large-scale organic synthetic process, ongoing research focuses on the development of metal-free catalytic systems. Notably, selective hydroboration of alkynes has been achieved in several cases using various metal-free catalytic systems. An important report by Jin et al. was published in 2014, which dealt with benzoic acid derivatives as potential catalysts for hydroboration. Notably, the authors demonstrated that internal alkynes substituted with Bpin (82) reacted efficiently to afford the synthetically 
important 1,1-diborylalkene 83 (Scheme 24). ${ }^{[96]}$ This catalytic system applies to a wide range of alkynes, yielding the expected syn-hydroborated product with exclusive selectivity.

(a) Piers' borane-catalyzed hydroboration of alkyne (Stephan, 2016)

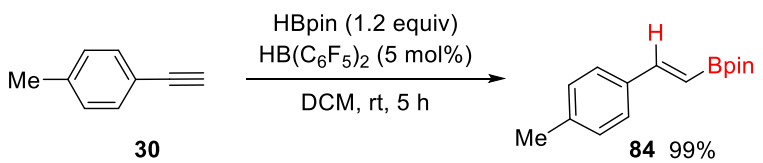

(b) $B\left(C_{6} F_{5}\right)_{3}$-catalyzed hydroboration of alkyne (Melen, 2017)

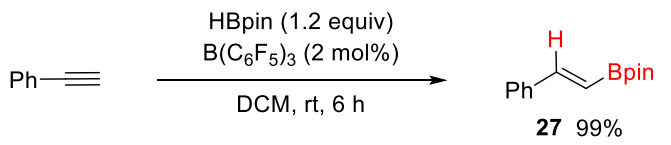

(c) Proposed mechanism of Piers' borane-catalyzed hydroboration of alkyne (Stephan, 2016)

$$
=\frac{\mathrm{HB}\left(\mathrm{C}_{6} \mathrm{~F}_{5}\right)_{2}}{\longrightarrow}
$$

Scheme 25. (a) and (b) Borane-catalyzed syn-selective hydroboration of alkynes and (c) its mechanistic aspect.

In 2016, Stephan et al. proposed the use of Piers' borane as a catalyst in the syn-selective hydroboration of internal and terminal alkynes (Scheme 25a). ${ }^{[97]}$ Recently, Melen et al. showed that $\mathrm{B}\left(\mathrm{C}_{6} \mathrm{~F}_{5}\right)_{3}$ also can be used for the same transformation, affording synselective products exclusively (Scheme $25 \mathrm{~b}$ ). ${ }^{[98]}$ The mechanistic aspects of such borane-catalyzed alkyne hydroboration reactions are interesting. In the case of the former catalytic reaction, Piers' borane $\left(\mathrm{HB}\left(\mathrm{C}_{6} \mathrm{~F}_{5}\right)_{2}\right)$ acts as a pre-catalyst, which generates an unsymmetrical gem-diborylated species (86) through the concerted hydroboration of alkynes, as shown in Scheme 25c. This syn-1,2-hydroborated species (86) serves as a catalyst, which at first enables the electrophilic activation of alkynes, to form $\sigma$-complex 87. Subsequently, concerted hydroboration of 87 proceeds in a syn-fashion via $\mathbf{8 8}$. Thus, product release occurs along with regeneration of the active catalyst 86.

Another metal-free catalytic hydroboration system, which deals with the use of $\mathrm{PhI}(\mathrm{OAc})_{2}$ as a catalyst, has been reported. Notably, various terminal alkynes were hydroborated in good yields with excellent regioand stereoselectivity (Scheme 26a). ${ }^{[99]}$ A plausible mechanism for $\mathrm{PhI}(\mathrm{OAc})_{2}-$ catalyzed hydroboration is given in Scheme 26b. At first, the intermediate $\mathbf{9 0}$ is generated with the reaction between $\mathrm{B}_{2}$ pin $_{2}$ and $\mathrm{NaO}^{t} \mathrm{Bu}$, which when reacted with the iodonium reagent (89) produces the key intermediate (91) containing the I-B bond. Subsequently, the addition of iodonium borane species (91) to alkynes proceeds in a syn-fashion to afford the intermediate $\mathbf{9 2}$. Finally, the target syn-hydroborated product is released in the presence of ethanol as a hydrogen donor reagent. Very recently, a metal-catalyst free electrochemical hydroboration of terminal alkyne was reported by Jubault and Poisson et al (Scheme 26c). ${ }^{[100]}$ The use of ${ }^{n} \mathrm{Bu}_{4} \mathrm{NBF}_{4}$ as an electrolyte in $\mathrm{MeOH}$, stainless steel electrodes (SSE) at both cathode and anode with a current of $10 \mathrm{~mA}$ and a total charge of $2 \mathrm{~F}^{\mathrm{mol}}{ }^{-1}$ were crucial for achieving exclusive syn-hydroborated product in excellent yield.

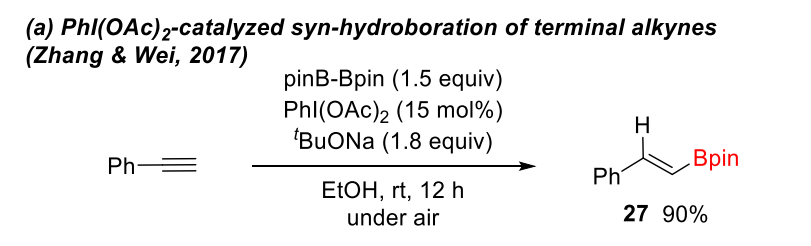

(b) Proposed mechanism for Phl(OAc) ${ }_{2}$-catalyzed syn-hydroboration of terminal alkynes (Zhang \& Wei, 2017)

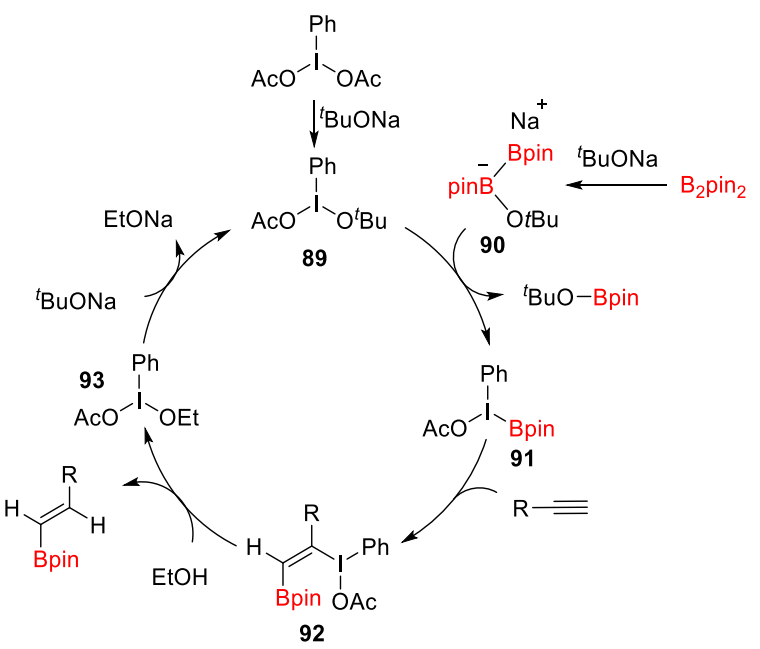

(c) Electrochemical syn-hydroboration of terminal alkyne (Jubault \& Poisson, 2021)

$$
\text { (+) SST }
$$

Scheme 26. (a) $\mathrm{PhI}(\mathrm{OAc})_{2}$ as a catalyst in the syn-selective hydroboration of terminal alkynes and (b) its mechanistic aspects. (c) Metal catalyst-free electrochemical synhydroboration of terminal alkyne. 


\section{3. anti-Hydroboration of alkynes}

As already mentioned, hydroboration of alkynes is of high significance as it provides a reliable and straightforward means of synthesizing synthetically important alkenyl borane compounds. Hence, obtaining a single isomer as a product of hydroboration of an alkyne is important. Notably, compared to the occurrence of syn-hydroboration products, anti-hydroboration is rare, due to the synselective nature of hydroboration and relative thermodynamic instability of the Z-isomer (in the case of a product generated from terminal alkynes). Hence, non-classical anti-hydroboration of alkynes has been mainly reported in a transition metal catalytic system. In this section, we summarize reported catalytic protocols for the anti-hydroboration of alkynes, along with their mechanistic aspects.

\subsection{Transition metal-catalyzed hydroboration}

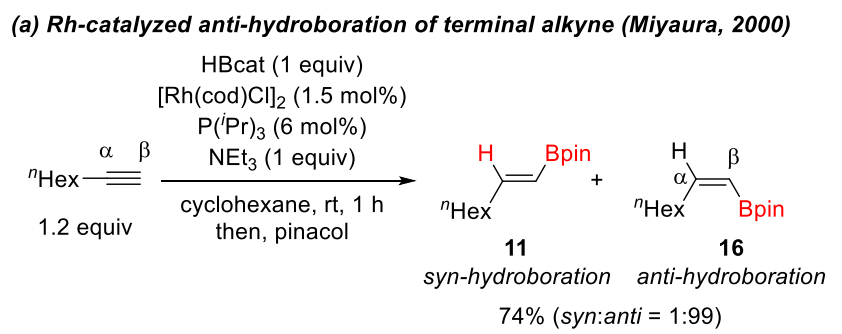

(b) Ir-catalyzed anti-hydroboration of terminal alkyne (Miyaura, 2000)

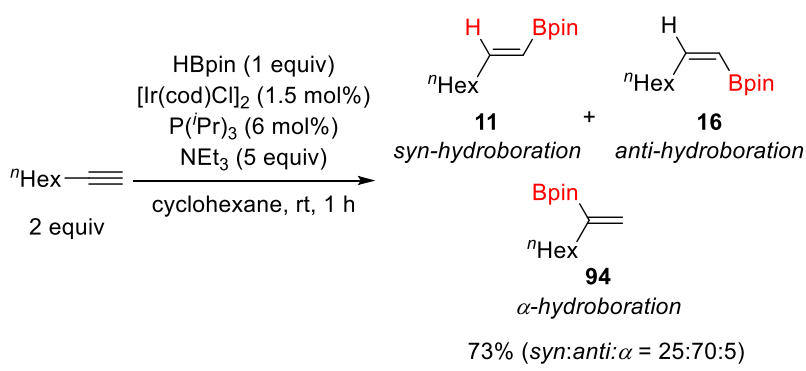

(c) Rh-catalyzed anti-hydroboration of alkyne (Carbo \& Fernandez, 2012)

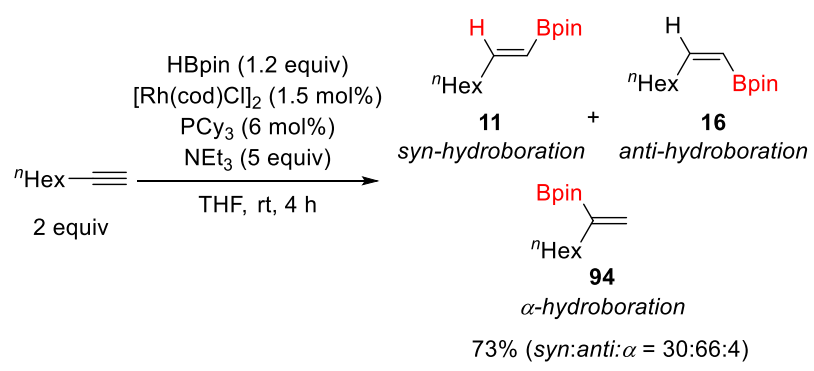

Scheme 27. (a) and (c) Rh- or (b) Ir-catalyzed antihydroboration of terminal alkynes.

The first report of anti-hydroboration of terminal alkynes was published by Miyaura et al. in 2000 using rhodium (Scheme 27a) or iridium (Scheme 27b) as a catalyst. ${ }^{[101]}$ Excellent anti-selectivity was obtained in the Rh-catalytic system; however, the Ir-catalyzed reaction displayed only moderate selectivity. Using the Rh-catalytic system, several terminal alkynes were selectively hydroborated to afford the corresponding Z-alkenyl boranes. A similar catalytic study was reported by the Carbo and Fernandez group (Scheme $27 \mathrm{c}),{ }^{[102]}$ although poor selectivity was noted, compared to the earlier report. In the study by Miyaura et al., the authors carried out a deuterium-labeling experiment using 1-d $d_{1}-1$-octyne and found, surprisingly, that in the final compound (16), the deuterium atom had shifted to the $\alpha$-position. Based on this observation, the authors proposed a nonconventional mechanism, in which an oxidative addition of the terminal $\mathrm{C}-\mathrm{H}$ bond to the metal center occurred, giving a metal hydride species (95). Subsequently, metal vinylidene complex 96 was formed through a hydrogen-shifting mechanism, followed by oxidative addition of HBpin to the metal center $(\mathbf{9 6} \rightarrow \mathbf{9 7}), 1,2$-migration of boryl group to $\alpha$ carbon $(\mathbf{9 7} \rightarrow \mathbf{9 8})$, and reductive elimination $(\mathbf{9 8} \rightarrow[\mathrm{M}])$ to generate the product (Scheme 28). ${ }^{[101]}$ The proposed mechanism was also supported by density functional theory (DFT) calculations. ${ }^{[102]}$

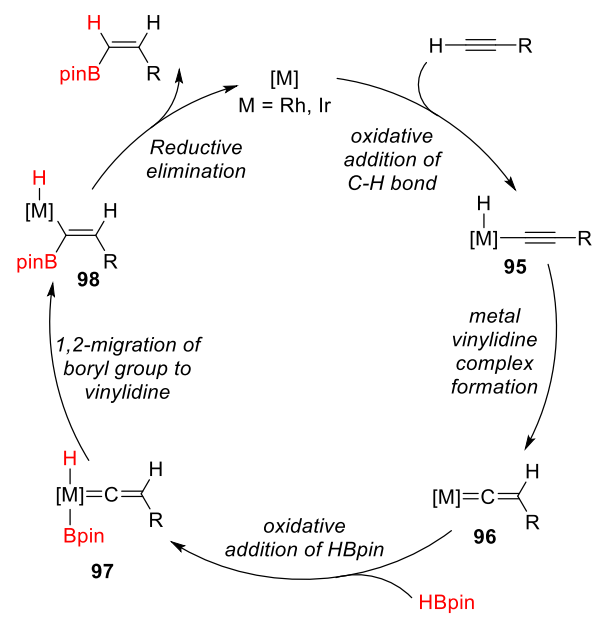

Scheme 28. A plausible mechanism for Rh- or Ir-catalyzed non-conventional anti-hydroboration of terminal alkynes (Miyaura, 2000). 
(a) Ru-catalyzed non-conventional anti-hydroboration of terminal alkyne (Leitner, 2012)

$$
\text { toluene, } \mathrm{rt}, 24 \mathrm{~h}
$$
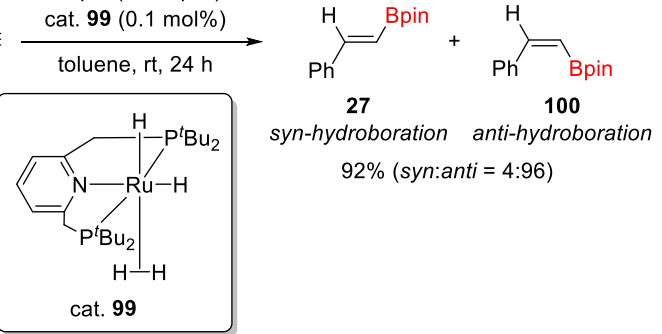

(b) Ru-catalyzed anti-hydroboration of internal alkyne (Furstner, 2013)

HBpin ( 1.2 equiv)

$\left[\mathrm{Ru}\left(\mathrm{CH}_{3} \mathrm{CN}\right)_{3} \mathrm{Cp}^{*}\right] \mathrm{PF}_{6}$

$$
\begin{aligned}
& \left.\left.\left.{ }^{n} \mathrm{Bu}={ }^{n} \mathrm{Bu} \frac{(5 \mathrm{~mol} \%)}{\mathrm{DCM}, \mathrm{rt}, 1 \mathrm{~h}}{ }_{{ }^{n} \mathrm{Bu}}\right\rangle^{\mathrm{H}}\right\rangle_{n_{\mathrm{Bu}}}^{\mathrm{Bpin}}+{ }_{{ }_{\mathrm{Bu}}}\right\rangle_{\mathrm{Bpin}}^{{ }^{n} \mathrm{Bu}} \\
& 101102 \\
& \text { syn-hydroboration anti-hydroboration } \\
& \text { 89\% (syn:anti }=3: 97 \text { ) }
\end{aligned}
$$

(c) Ru-catalyzed anti-hydroboration of terminal alkyne (Mutoh \& Saito, 2019)
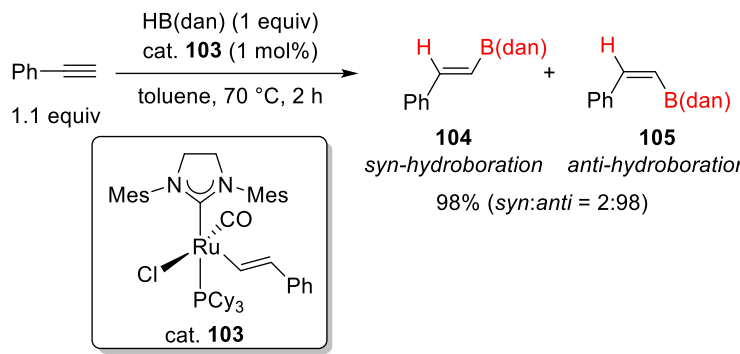

syn-hydroboration anti-hydroboration

$98 \%$ (syn:anti $=2: 98$ )

(d) Ru-catalyzed anti-hydroboration of secondary propargyl alcohols (Furstner, 2019)

$$
\begin{aligned}
\begin{array}{c}
106 \\
\text { syn-hydroboration anti-hydroboration }
\end{array} \\
\mathrm{R}=\mathrm{Ac} ; 64 \% \text { (syn:anti }=0: 100) \\
\mathrm{R}=\mathrm{MOM} ; 87 \%(\text { syn:ant } i=0: 100) \\
\mathrm{R}=\mathrm{TES} ; 83 \% \text { (syn:anti }=0: 100) \\
\mathrm{R}=\mathrm{TIPS} ; 94 \% \text { (syn:anti }=8: 92)
\end{aligned}
$$

Scheme 29. (a)-(d) Ru-catalyzed anti-hydroboration of alkynes.

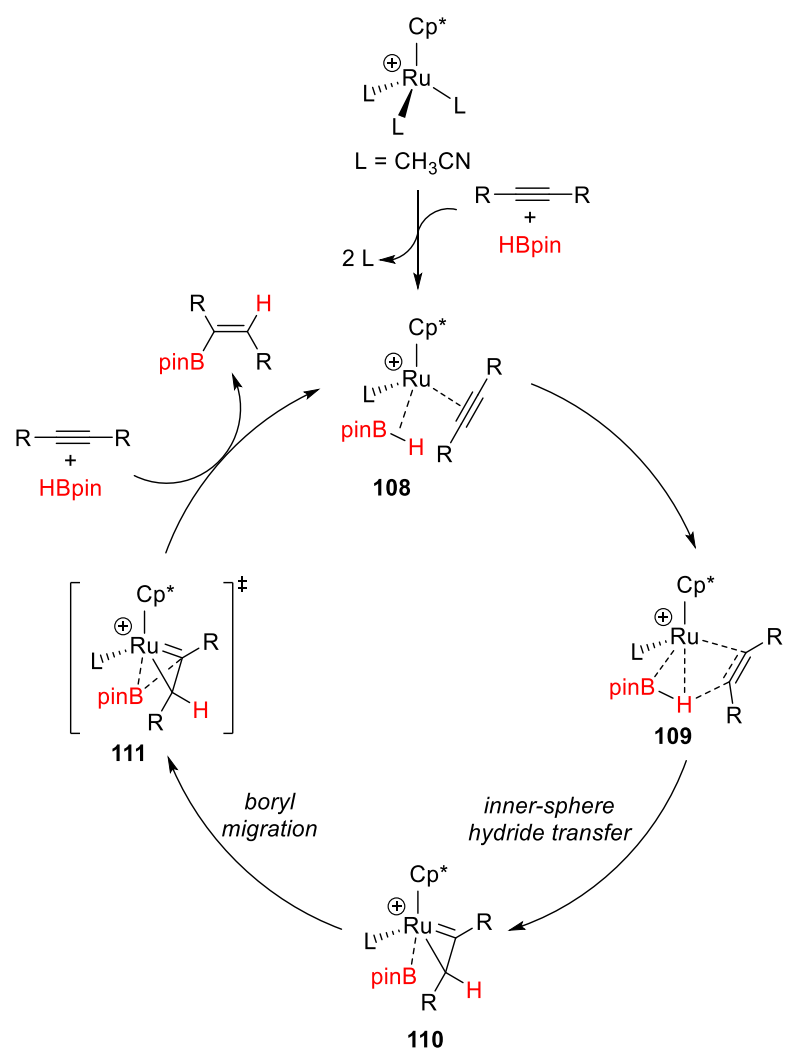

Scheme 30. A most plausible mechanism for Ru-catalyzed anti-hydroboration of internal alkynes (Zhang, Chung, and $\mathrm{Wu}, 2017)$.

In 2012, Leitner et al. introduced a ruthenium hydride pincer complex that efficiently catalyzed the unorthodox anti-hydroboration of terminal alkynes with a minimum catalyst loading $(0.1 \mathrm{~mol} \%)$ (Scheme 29a). ${ }^{[103]}$ Based on the detection of plausible intermediates, it was proposed that rearrangement of coordinated alkynes to Z-vinylidene via hydrogen shifting is a key step in the catalytic cycle, as shown in the case of the Rh-catalytic system (Scheme 28). One year after this report, Furstner et al. reported the antiselective hydroboration of internal alkynes using $\mathrm{Ru}$ complex $\left[\mathrm{Cp} * \mathrm{Ru}(\mathrm{MeCN})_{3}\right]^{+}$as the catalyst (Scheme 29b). ${ }^{[104]}$ This report was the first to achieve transselective hydroboration of internal alkynes. A range of alkynes is selectively hydroborated in anti-fashion. In the report, the authors hypothesized that the alkyne moiety serves as a four-electron donor and holds a bonding interaction with the $\mathrm{Ru}$ center, which facilitates an inner-sphere hydride transfer to form the metallacyclopropene intermediate $\mathbf{1 1 0}$ (Scheme 30). ${ }^{[104]}$ Subsequently, facile reductive boryl migration via transition state $\mathbf{1 1 1}$ helped to afford the transselective product. Detailed DFT calculations suggested that the anti-selectivity was determined in the oxidative hydrogen migration step, which is a ratelimiting step, and selectively formed the metallacyclopropene intermediate 110. ${ }^{[105]}$ Recently, the Mutoh and Saito group reported the hydroboration of terminal alkynes catalyzed by $N$-heterocycliccarbene-ligated ruthenium complex 103, using 
$\mathrm{HB}$ (dan) as a hydroborating reagent (Scheme 29c). ${ }^{[106]}$ A variety of terminal alkynes are selectively hydroborated to produce the desired anti-selective product. Mechanistic studies suggest that a distinctly different mechanism occurs, which involves the insertion of the alkyne into a Ru-B bond, followed by rapid isomerization to form a more stable component via a metallacyclopropene intermediate. This mechanism is in line with the proposed mechanism reported for the anti-hydroboration of internal alkynes (Scheme 30). Furstner et al. further extended the Rucatalyzed anti-hydroboration study to secondary propargyl alcohol derivatives, which in some cases provided the trans-hydroborated product exclusively (Scheme 29d). ${ }^{[107]}$
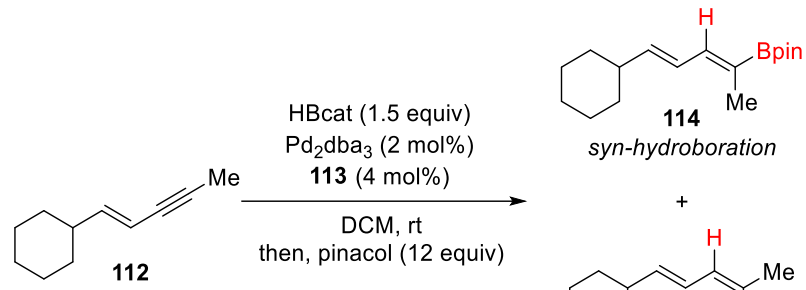

syn-hydroboration

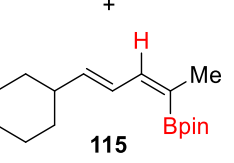

anti-hydroboration

$92 \%$ (syn:anti $=4: 96)$

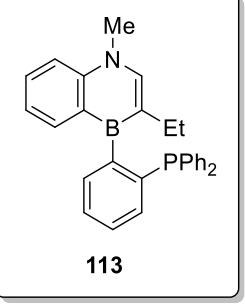

Scheme 31. 1,4-azaborine-based Phosphine ligated palladium-catalyzed anti-hydroboration of 1,3-enynes (Liu, 2016).

Liu et al. introduced a new type of monobenzofused 1,4-azaborine phosphine ligand (113), which uniquely supports a Pd complex for stereoselective anti-hydroboration of 1,3-enynes (Scheme 31). ${ }^{[108]}$ A range of internal and terminal 1,3enynes are selectively anti-hydroborated to deliver dienylboronates (such as 115). Detailed mechanistic exploration in terms of computational studies was carried out, showing a complicated pathway (different from the conventional mechanism) in which the coordination of the conjugated alkene to the metal center was recognized as a crucial event. ${ }^{[109]}$

Along with precious metal catalysts, a few novel complexes of earth-abundant first-row transition metals have also been used as catalysts. In 2011, Yun et al. briefly reported the anti-hydroboration of aryl and bulky tert-butyl group substituted internal alkyne (3,3-dimethylbut-1-yn-1-yl)benzene) to yield the corresponding product 116 (Scheme 32a). They realized that sterically demanding ${ }^{t} \mathrm{Bu}$ group and phenyl substitutions are crucial to achieving antihydroboration. ${ }^{[63]}$ Later, the same group reported a $\mathrm{Cu}-$ catalyzed system with a combination of copper(I)-thiophene-2-carboxylate (CuTC) and DPEphos ligand to achieve the anti-hydroboration of terminal alkynes using $\mathrm{HB}(\mathrm{dan})$ (Scheme $32 b) .{ }^{[110]}$ (a) Cu-catalyzed anti-hydroboration of internal alkyne having sterically demanding tBu group (Yun, 2011)

$\mathrm{B}_{2} \mathrm{pin}_{2}$ (1.1 equiv)

$\mathrm{CuCl}(5 \mathrm{~mol} \%)$

$\mathrm{P}(p \text {-tolyl })_{3}(6 \mathrm{~mol} \%)$

$\mathrm{Ph}={ }_{\mathrm{Bu}}^{{ }_{\mathrm{B}} \mathrm{Mu}} \stackrel{\mathrm{MeOH}(2 \text { equiv) }}{\mathrm{THF}, 50{ }^{\circ} \mathrm{C}, 20 \mathrm{~h}}$

$11691 \%$

(b) CuTC/DPEphos-catalyzed anti-hydroboration of terminal alkyne (Lee \& Yun, 2016)

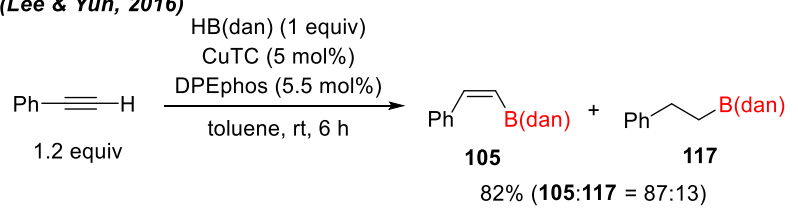

(c) SIPr-CuCl-catalyzed syn-hydroboration of terminal alkyne

(Lee \& Yun, 2016)

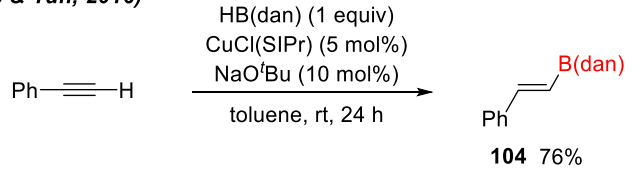

(d) Mechanistic aspect for Cu/ligand-catalyzed hydroboration of alkyne

(Lee \& Yun, 2016) L + CuTC + HB(dan)

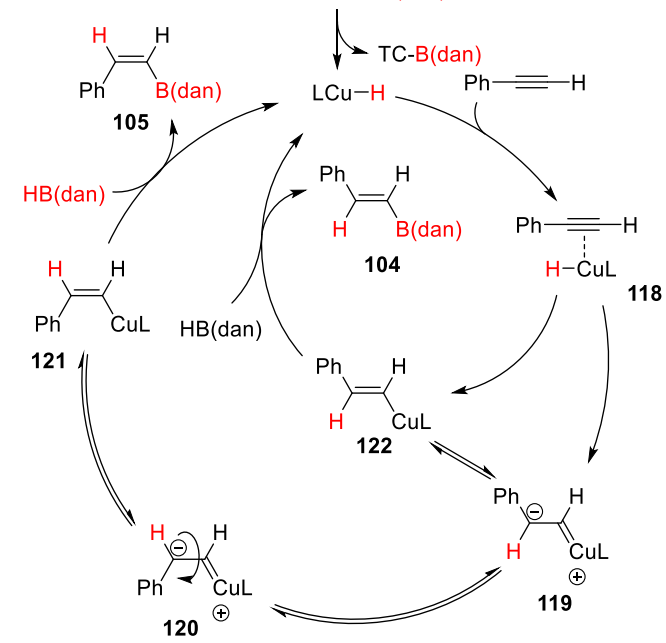

Scheme 32. (a) and (b) Cu-catalyzed anti-hydroboration of internal and terminal alkynes. (c) syn-Hydroboration reaction using a similar $\mathrm{Cu}$-catalytic system. (d) A plausible mechanism for $\mathrm{Cu}$-catalyzed anti-hydroboration.

It is also notable that when strong $\sigma$-donating ligands, such as the bulky $N$-heterocyclic carbene ligand (SIPr), were used, syn-hydroboration proceeded exclusively (Scheme 32c). ${ }^{[110]}$ For the unusual antihydroboration reaction, the author proposed a plausible mechanism, as shown in Scheme 32d. At first, a copper-hydride species was generated, which upon reaction with alkyne, formed the intermediate (119). Subsequent $\mathrm{C}-\mathrm{C}$ bond rotation via $\mathbf{1 2 0}$ generated 121. In the last step, $\sigma$-bond metathesis of the organocopper intermediate and borane provided the desired product, either $\mathbf{1 0 5}$ (via 121) or $\mathbf{1 0 4}$ (via 122). The selectivity of the product was determined by the ligand (NHC or phosphine) used. Another important factor for determining the stereoisomer is the hydroborane reagent used, since $\mathrm{HB}($ dan) prefers the alkenyl copper intermediate in which the phenyl group lies cis with $\mathrm{Cu}$, to avoid the steric interaction (as shown in 121). ${ }^{[110]}$ 
(a) Co-catalyzed anti-hydroboration of terminal alkyne (Chirik, 2015)

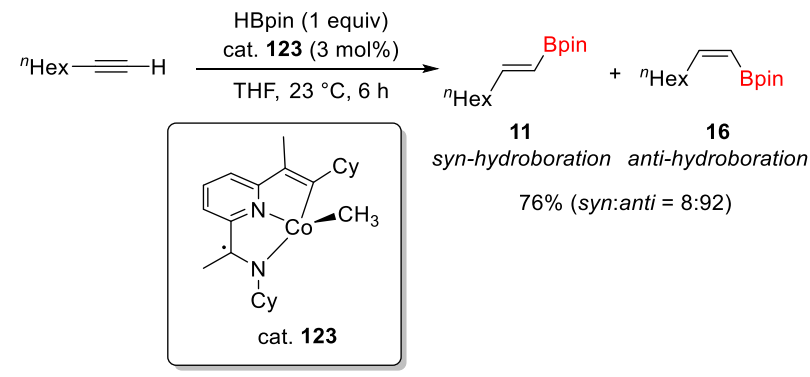

(b) Mechanistic consideration for Co-catalyzed anti-hydroboration of terminal alkyne (Chirik, 2015)

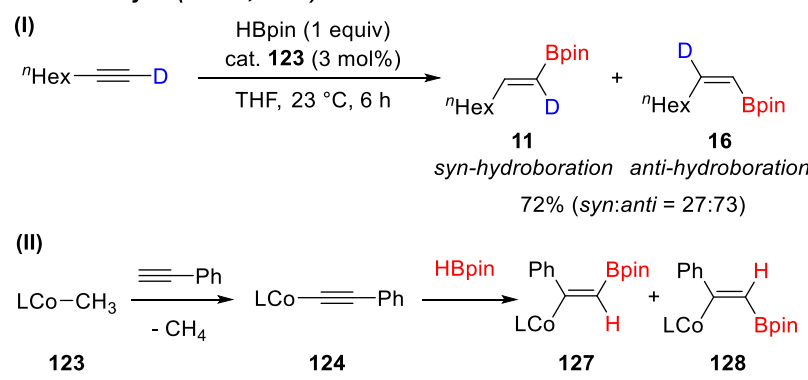

(c) Proposed mechanism for Co-catalyzed anti-hydroboration of terminal alkyne (Chirik, 2015)
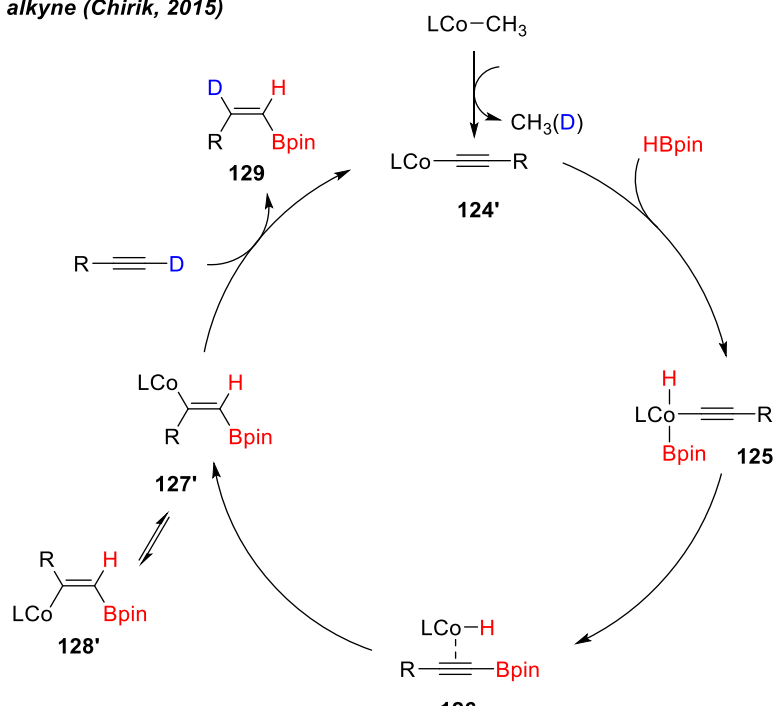

Scheme 33. (a) Co-catalyzed anti-hydroboration of terminal alkynes, (b) its mechanistic studies, and (c) plausible mechanism.

anti-Selective hydroboration of terminal alkynes was reported by Chirik et al. using a bis(imino)pyridine ligated Co complex (123) (Scheme 33a). ${ }^{[11]} \mathrm{A}$ unique and distinct reaction pathway was proposed for this reaction based on deuterium studies and control experiments (Scheme 33b). In the deuterium studies, it was observed that the terminal Datom in 1-d $d_{1}-1$-octyne shifted to the 2-position in product 16 (Scheme 33b-I). However, in a controlled reaction between organocobalt compound $\mathbf{1 2 4}$ and HBpin, 127 and $\mathbf{1 2 8}$ were generated. It was detected that in $\mathbf{1 2 7}$ or $\mathbf{1 2 8}$, the carbon bound to the cobalt is phenyl substituted, while the distal carbon is bound to the Bpin (Scheme 33b-II), which is completely unlike the previously reported mechanism via vinylidene $(\mathbf{9 7} \rightarrow \mathbf{9 8}$, as shown in Scheme 28). Based on these studies, the authors proposed that following the formation of cobalt acetylide (124'), oxidative addition of HBpin followed by reductive elimination resulted in the formation of alkynylboronate $\mathbf{1 2 6}$ (Scheme 33c). Subsequently, syn-hydrometallation resulted in the vinylic cobalt intermediate 127', which reacted with terminal alkynes to release the product and active cobalt species. ${ }^{[11]}$ This catalytic cycle also supports the observation that the D-atom in product $\mathbf{1 6}$ came from another molecule of alkyne.

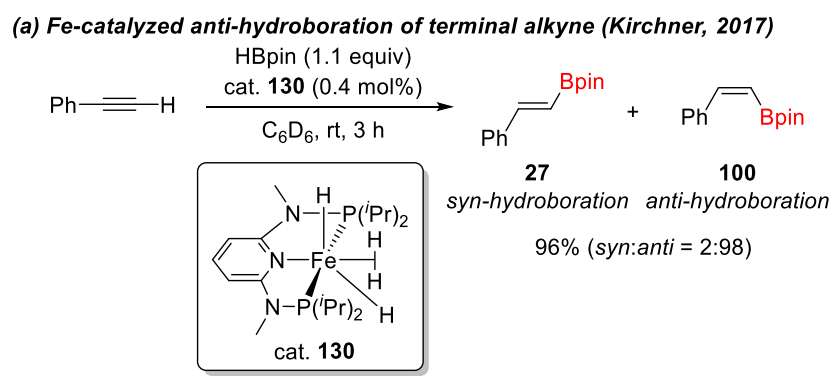

(b) Fe-catalyzed anti-hydroboration of terminal alkyne (Ruiter, 2020)
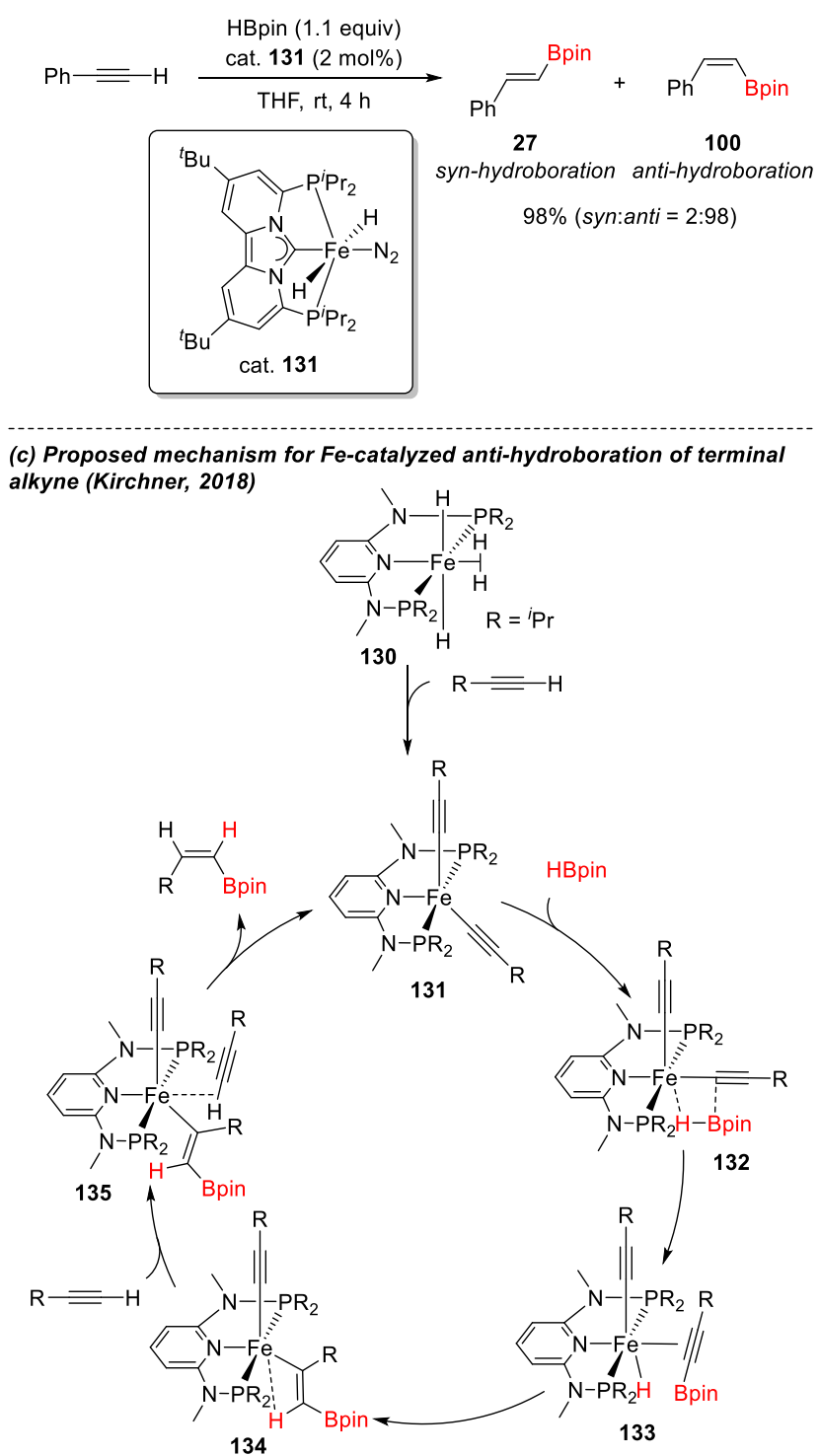

Scheme 34. (a) and (b) Fe-catalyzed anti-hydroboration of terminal alkynes. (c) A plausible mechanism for $\mathrm{Fe}-$ catalyzed anti-hydroboration. 
In 2017, a tridentate PNP pincer-type ligand ligated polyhydride Fe-complex (130) was introduced by Kirchner and coworkers for the stereoselective antihydroboration of terminal alkynes (Scheme 34a). ${ }^{[12]}$ Their protocol covered a range of terminal alkynes to affords corresponding $Z$-alkenes in excellent yields and selectivity. In a successive report, the authors proposed a reaction mechanism (Scheme 34c) ${ }^{[113]}$ based on computational studies and experimental evidence, which followed a pattern similar to the Cocatalytic system (as shown in Scheme 33c). Very recently, Ruiter et al. developed an $N$-heterocyclic carbene-based PCP ligand ligated trans-dihydride iron complex (131), which efficiently catalyzed the antihydroboration of terminal alkynes to provide Zalkenylborane compounds (Scheme 34b). ${ }^{[14]}$

\subsection{Metal-free catalytic anti-hydroboration}

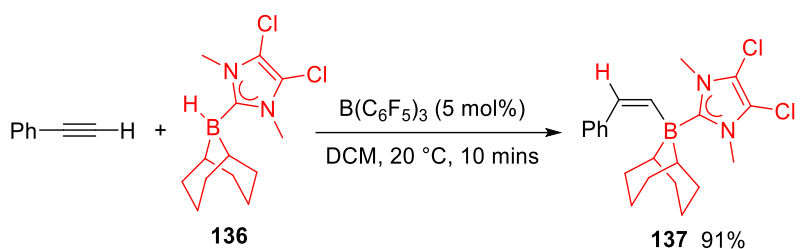

Scheme 35. $\mathrm{B}\left(\mathrm{C}_{6} \mathrm{~F}_{5}\right)_{3}$-catalyzed anti-hydroboration of the terminal alkyne (Ingleson, 2016).

Although anti-hydroboration of alkynes is a rare transformation compared to the syn variant, there are a few reports in which borane or phosphines were used as efficient catalysts for this selective transformation. Incidentally, the first transition metal-free transhydroboration was reported by Ingleson et al. in 2016 using a catalytic amount of $\mathrm{B}\left(\mathrm{C}_{6} \mathrm{~F}_{5}\right)_{3}$ and $N$ heterocyclic carbene coordinated hydroborane 136 (Scheme 35). ${ }^{[15]}$ Notably, various terminal alkynes were selectively hydroborated in an anti-fashion to yield the corresponding product in excellent yield in a very short reaction time. Apart from the use of $\mathrm{B}\left(\mathrm{C}_{6} \mathrm{~F}_{5}\right)_{3}$ as a catalyst in the anti-hydroboration reaction, phosphine also displayed impressive reactivity as a catalyst in the same transformation. Three parallel independent reports, published by the working groups of Ohmiya/Sawamura, ${ }^{[116]}$ Vilotijevic, ${ }^{[117]}$ and Santos ${ }^{[118]}$ presented an attractive catalytic system in which a trialkylphosphine serves as an organocatalyst in the anti-hydroboration of alkynoic acid derivatives such as alkynoates (138), alkynylamides (139), adjacent pyrimidine (140), benzoxazole (141), benzothiazole (142), and benzoimidazole (143) group containing alkynes (Scheme 36a). It was hypothesized that the reaction started with the conjugate addition of phosphine to the electron-deficient alkyne moiety with the assistance of Lewis acidic activation of carbonyl or azole groups by HBpin (as shown in 144, Scheme 36b). Later, hydride transfer to the allene moiety occurred, to form an ylide intermediate 145. Subsequently, the resonance of the conjugated system, followed by $\mathrm{C}-\mathrm{C}$ bond rotation, easily led to a suitable position for the ylide carbon to attack the $\mathrm{B}$ center to form a five-membered intermediate (149). In the last step, elimination of phosphine and cleavage of $\mathrm{B}-\mathrm{X}$ bond afforded the anti-hydroborated product. The concerted nature of the final phosphine elimination step is mainly responsible for obtaining high trans-selectivity. ${ }^{[16]}$

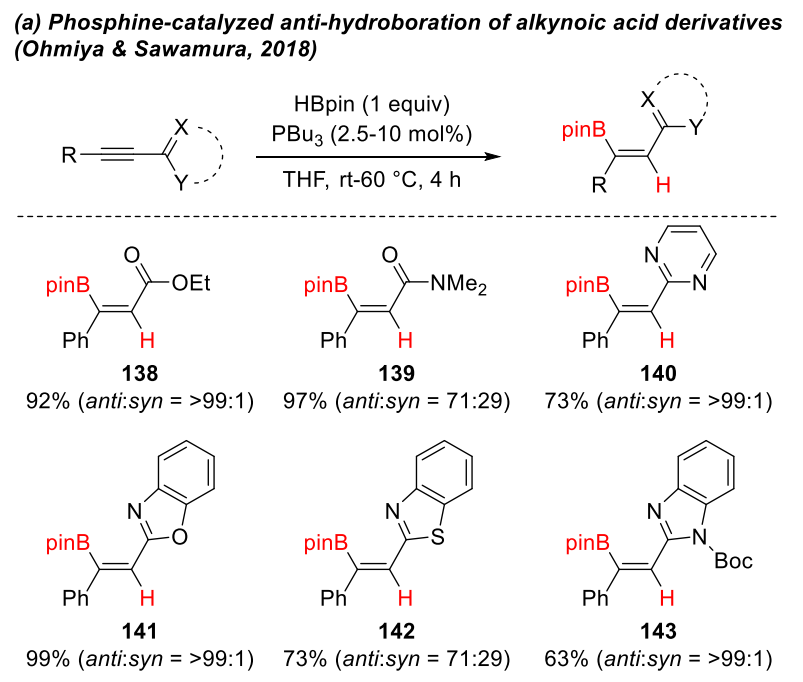

(b) Proposed mechanism for phosphine-catalyzed anti-hydroboration of alkyne (Ohmiya \& Sawamura, 2018)

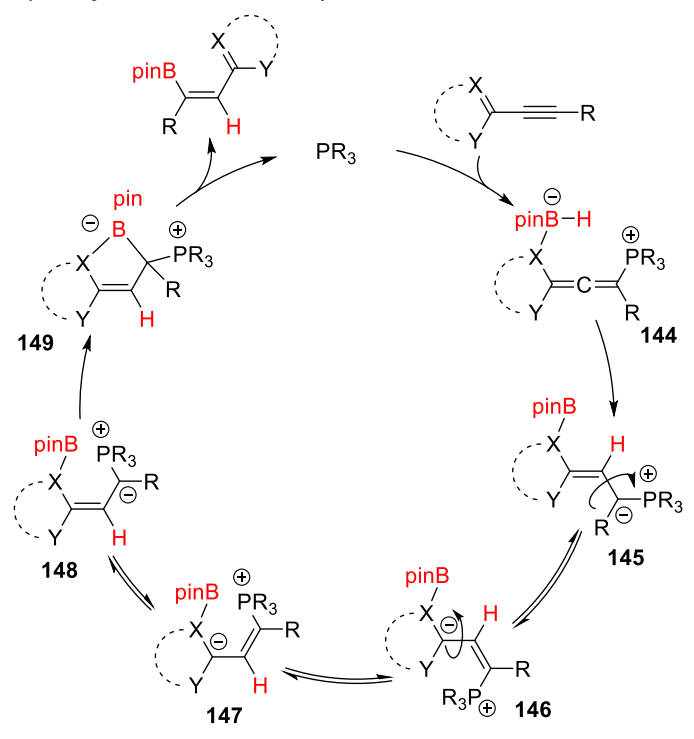

Scheme 36. (a) Phosphine-catalyzed anti-hydroboration of various alkynoic acid derivatives and (b) the plausible catalytic pathway.

\section{4. a-Selective hydroboration of terminal alkynes}

Considering the significance of vinyl borane compounds, several strategies have been employed to obtain the selective hydroboration product either via cis- or trans addition, which has already been discussed in the previous sections in detail. Reports discussed in previous sections dealt with the formation of $\beta$-borylated products when terminal alkyne was 
used as a substrate. However, obtaining $\alpha$-vinyl boronated compounds via hydroboration of terminal alkynes is significantly limited. Methods reported to date for obtaining $\alpha$-selective hydroboration are limited to copper catalytic systems. ${ }^{[119-123]}$ The pioneering report in this regard was published by Hoveyda et al. using $N$-heterocyclic carbene-ligated copper complexes (Scheme 37). Specifically, various propargyl alcohol (153 and 154) and amine-derived terminal alkynes (155 and 156) were reacted smoothly to afford the corresponding $\alpha$-borylated alkenes. Arylsubstituted terminal alkyne is also a suitable substrate for this transformation, giving the desired product 157 in high yield and with good selectivity. Based on mechanistic studies, it was anticipated that the desired $\alpha$-selectivity arises from the electronic and structural properties of the NHC ligand. Hence, aryl-substituted NHC-ligated copper complexes (150 and 151) were used for obtaining $\alpha$-selectivity, whereas, adamantyl group substituted NHC-Cu complex (152) provided $\beta$ borylated alkene (158) when phenylacetylene was used as a substrate (Scheme 37). ${ }^{[119]}$
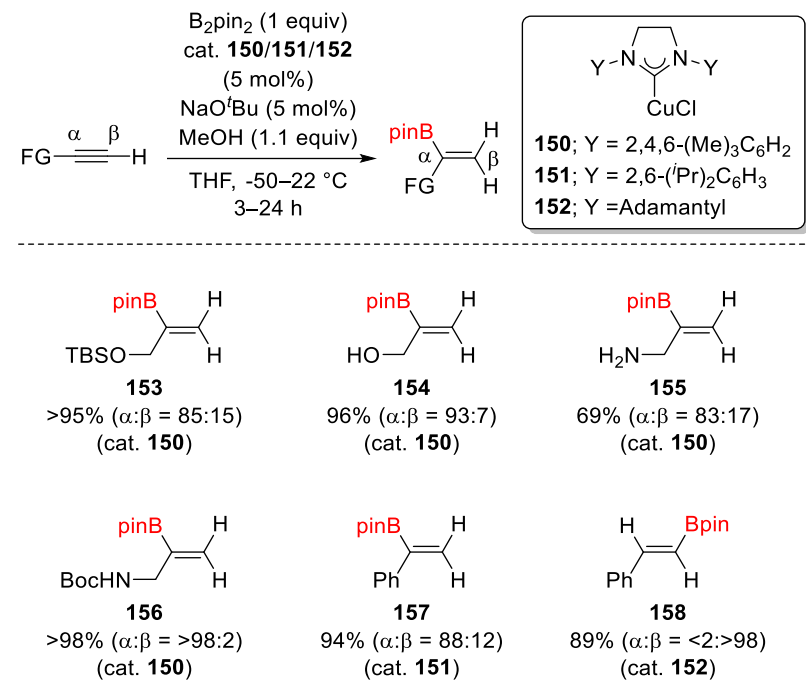

Scheme 37. NHC-Cu-catalyzed $\alpha$-selective hydroboration of terminal alkynes (Hoveyda, 2011).

A few years later, Yoshida et al. introduced masked diborane [(pin)B-B(dan)] as a reagent for the $\mathrm{Cu}$ NHC-catalyzed hydroboration of terminal alkynes, which eventually afforded the $\alpha$-borylated alkene (94) even when alkyl-substituted terminal alkyne was used as a substrate (Scheme 38a). ${ }^{[121]}$ Notably, this protocol showed impressive selectivity and displayed a substrate generality, since alkyl-, aryl-, heteroatom-, and silyl-substituted terminal alkynes reacted smoothly to provide the corresponding $\alpha$-borylated product. The authors hypothesized that the use of masked borane is the key factor, since the Lewis acidity of $\mathrm{B}(\mathrm{dan})$ moiety in $\mathbf{1 5 9}$ is weakened, and the interaction between alkyne moiety and boron center is negligible. (This interaction is one of the factors for $\beta$ selective addition through a more stable cationic transition state.) Therefore, a steric factor is the only remaining factor for determining position selectivity. Due to the bulkiness of the NHC ligand, it was expected that 160 would be preferred over 160' as an intermediate, and hence the $\alpha$-selective product forms almost exclusively (Scheme 38b). ${ }^{[121]}$

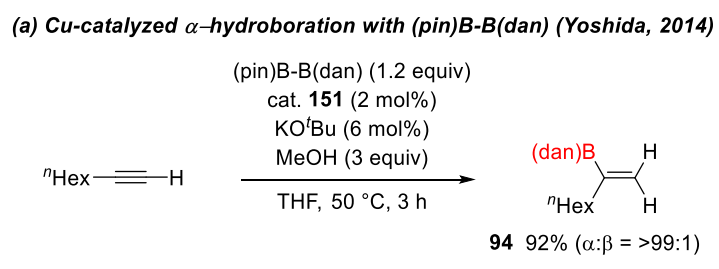

(b) Proposed mechanism for Cu-catalyzed $\alpha$-hydroboration with (pin)B-B(dan) (Yoshida, 2014)

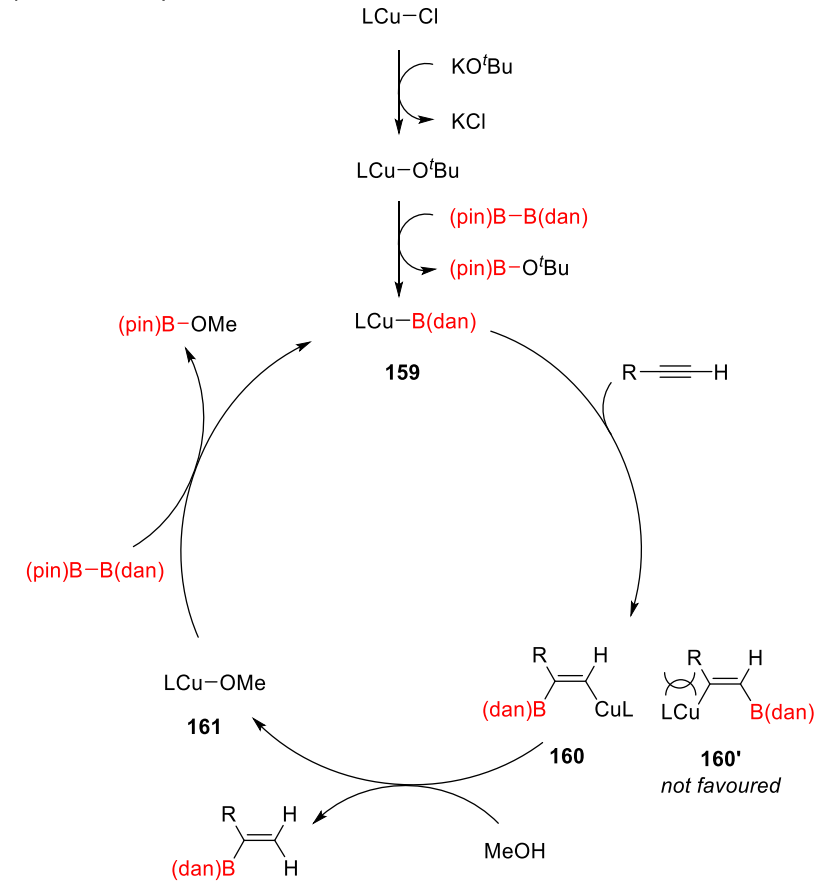

Scheme 38. (a) Cu-catalyzed $\alpha$-selective hydroboration of terminal alkynes using (pin)B-B(dan) and (b) its mechanistic aspects.

The Arrayas and Carretero research group also studied the $\alpha$-selective hydroboration of propargylic functional group-derived terminal alkynes (Scheme 39a). ${ }^{[120]}$ Notably, propargyl-substituted sulfone (162), alcohol (163), amine (156), and sulfide (164) derivatives provided the smooth formation of $\alpha$ selective products. As expected, the hydroboration of 2-propynylbenzene did not give up-to-the-mark selectivity (165), which suggested that the polar functional group substitution contributed to high $\alpha$ selectivity. Another important point to note is that a bulky phosphine ligand was used in this case to provide a suitable steric interaction to accomplish high selectivity. Moro et al. later developed a similar catalytic transformation, which can work in the $\mathrm{H}_{2} \mathrm{O}$ medium in the presence of a surfactant SPGS-550M (Scheme 39b). ${ }^{[122]}$ Recently, Jones and coworkers disclosed that $N$-heterocyclic carbene-ligated copper 
hydroxide $(\mathrm{Cu}(\mathrm{IPr})(\mathrm{OH}) ; \quad \mathrm{IPr}=1,3-\mathrm{bis}(2,6-$ diisopropylphenyl)-imidazol-2-ylidene) would work as a catalyst for $\alpha$-selective hydroboration of phenylacetylene in the absence of any additives (Scheme 39c). ${ }^{[123]}$ Although reports of $\alpha$-selective hydroboration of terminal alkynes are scarce compared to $\beta$-selective hydroboration, research on the former is expected to flourish more in the coming decades.

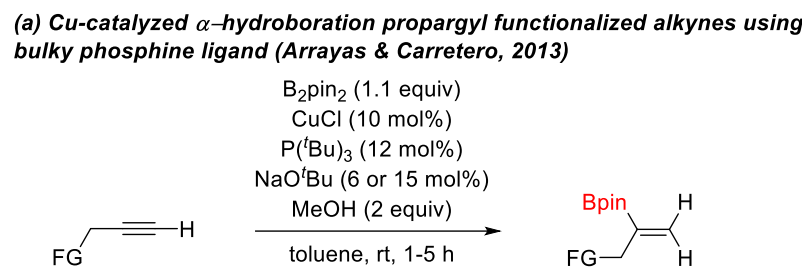

162; $\mathrm{FG}=\mathrm{SO}_{2} \mathrm{Ph} ; 80 \%(\alpha: \beta=>98:<2)$ 163; FG $=$ OAC; $70 \%(\alpha: \beta=>98:<2)$ 156; $\mathrm{FG}=\mathrm{NHBoc} ; 76 \%(\alpha: \beta=>98:<2)$ $164 ; \mathrm{FG}=\mathrm{SPh} ; 76 \%(\alpha: \beta=>98:<2)$ 165; $F G=P h ; 70 \%(\alpha: \beta=67: 33)$

(b) Cu-catalyzed $\alpha$-hydroboration propargyl functionalized alkynes in water (Moro, 2016)

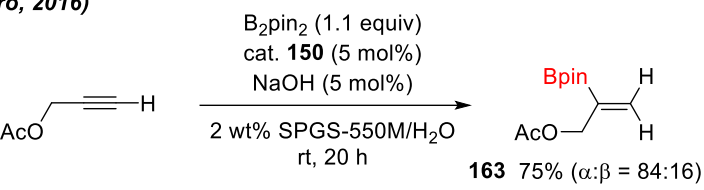

(c) NHC-Cu hydroxide as a catalyst for additive free $\alpha$-hydroboration terminal alkynes (Jones, 2019)

$$
\begin{aligned}
& \mathrm{Ph}=\mathrm{H} \quad \stackrel{\mathrm{Cu(IPr})(\mathrm{OH})(0.5 \mathrm{~mol} \%)}{\text { neat, } \mathrm{rt}, 24 \mathrm{~h}} \\
& 157 \text { quant. } \\
& \text { Note: exclusively } \\
& \alpha \text {-hydroborated product }
\end{aligned}
$$

Scheme 39. Various catalytic systems for $\mathrm{Cu}$-catalyzed $\alpha$ selective hydroboration of terminal alkynes.

\section{Conclusion and future direction}

In the past few decades, catalytic regioselective and stereoselective hydroboration of alkynes has been studied extensively in terms of reaction condition development, mechanistic exploration, and computational studies. Various catalytic systems have been employed from transition metal catalytic systems to alkali metal/alkaline earth metal, and Al-based catalytic systems. Even metal-free catalytic systems have been studied on several occasions to achieve selective hydroboration of alkynes. For readers to get an overview of the methods used in this selective transformation, this review summarizes reports of previous studies on the regioselective and stereoselective aspects of hydroboration. Three main types of selective hydroboration of alkynes are discussed in this review. (a) syn-Hydroboration, in which the addition of hydroborane proceeds via a cisprocess. This is the most studied process in alkyne hydroboration. (b) anti-Hydroboration, where hydrogen and boron moieties are added to an alkyne in a trans-fashion. Remarkably, a few pioneering reports have opened the window to this transformation and notable progress has been witnessed. (c) $\alpha$-selective hydroboration of terminal alkynes, which is still limited to the copper-catalytic system, was introduced by a pioneering report by Hoveyda et al. ${ }^{[119]}$ Despite much progress, further advancement in this field depends on certain factors. These are: (a) alkenyl borane compounds are widely used in the synthesis of complex molecules in multi-step processes. If hydroboration of an alkyne can be performed even in the presence of impurities (generated from the last synthetic step), it would be a step-change in the economy of the processes; (b) given the ongoing interest in the reaction under continuous flow, hydroboration of alkynes is a probable candidate for improving productivity; (c) further industrial application is possible through the development of a more mild, practical, environmentally benign, metalfree protocol, which can be sustained in the presence of air and water. Overall, it is expected that more research will be reported in the coming years in terms of the development of new catalytic systems. We expect that in the future more efforts will be undertaken to establish new strategies to exclusively achieve a single isomer in an alkyne hydroboration process. We hope that this comprehensive review will help researchers to gather new ideas and stimulate them in undertaking more research in this field.

\section{Data availability statement}

The data that support the findings of this study are openly available in references.

\section{References}

[1] A. Suzuki, Heterocycles 2010, 80, 15-43.

[2] A. Suzuki, Angew. Chem. Int. Ed. 2011, 50, 67236737.

[3] I. P. Beletskaya, F. Alonso, V. Tyurin, Coord. Chem. Rev. 2019, 385, 137-173.

[4] H. C. Brown, B. C. S. Rao, J. Am. Chem. Soc. 1956, 78, 2582-2588.

[5] H. C. Brown, B. C. S. Rao, J. Am. Chem. Soc. 1956 78, 5694-5695.

[6] R. Koster, Justus Liebigs Ann. Chem. 1958, 618, 31-43.

[7] A.-M. Carroll, T. P. O'Sullivan, P. J. Guiry, Adv. Synth. Catal. 2005, 347, 609-631.

[8] C. C. Chong, R. Kinjo, ACS Catal. 2015, 5, 32383259.

[9] K. Kuciński, G. Hreczycho, Green Chem. 2020, 22, 5210-5224.

[10] D. Hayrapetyan, A. Y. Khalimon, Chem. - An Asian J. 2020, 15, 2575-2587.

[11] A. D. Bage, K. Nicholson, T. Langer, S. P. Thomas, ACS Catal. 2020, 10, 13479-13486. 
[12] L. Mao, S. K. Bose, Adv. Synth. Catal. 2020, 362, 4174-4188.

[13] Z. Cheng, J. Guo, Z. Lu, Chem. Commun. 2020, 56, 2229-2239.

[14] V. B. Saptal, R. Wang, S. Park, RSC Adv. 2020, 10, 43539-43565.

[15] K. K. Das, S. Paul, S. Panda, Org. Biomol. Chem. 2020, 18, 8939-8974.

[16] J. Guo, Z. Cheng, J. Chen, X. Chen, Z. Lu, Acc. Chem. Res. 2021, 54, 2701-2716.

[17] G. I. Nikonov, ACS Catal. 2017, 7, 7257-7266.

[18] J. V. Obligacion, P. J. Chirik, Nat. Rev. Chem. 2018, 2, 15-34.

[19] J. Chen, J. Guo, Z. Lu, Chinese J. Chem. 2018, 36, 1075-1109.

[20] Z. Zuo, H. Wen, G. Liu, Z. Huang, Synlett 2018, 29, 1421-1429.

[21] M. L. Shegavi, S. K. Bose, Catal. Sci. Technol. 2019, 9, 3307-3336.

[22] W. Fan, L. Li, G. Zhang, J. Org. Chem. 2019, 84, 5987-5996.

[23] C. Nájera, I. P. Beletskaya, M. Yus, Chem. Soc. Rev. 2019, 48, 4515-4618.

[24] Y. Wen, C. Deng, J. Xie, X. Kang, Molecules 2019, 24, 101.

[25] H. Yoshida, ACS Catal. 2016, 6, 1799-1811.

[26] X. Wang, Y. Wang, W. Huang, C. Xia, L. Wu, ACS Catal. 2021, 11, 1-18.

[27] H. C. Brown, S. K. Gupta, J. Am. Chem. Soc. 1975, 97, 5249-5255.

[28] D. Mannig, H. Noth, Angew. Chem. Int. Ed. Engl. 1985, 24, 878-879.

[29] S. Makoto, Y. Nomoto, N. Miyaura, A. Suzuki, Tetrahedron Lett. 1989, 30, 3789-3792.

[30] C. Xue, S.-H. Kung, J.-Z. Wu, F.-T. Luo, Tetrahedron 2008, 64, 248-254.

[31] S. Pereira, M. Srebnik, Tetrahedron Lett. 1996, 37, 3283-3286.

[32] J. J. J. Juliette, D. Rutherford, I. T. Horváth, J. A. Gladysz, J. Am. Chem. Soc. 1999, 121, 2696-2704.

[33] T. Lee, C. Baik, I. Jung, K. H. Song, S. Kim, D. Kim, S. O. Kang, J. Ko, Organometallics 2004, 23, 45694575.

[34] B. M. Neilson, C. W. Bielawski, Organometallics 2013, 32, 3121-3128.

[35] C. N. von Hahmann, M. Talavera, C. Xu, T. Braun, Chem. - A Eur. J. 2018, 24, 11131-11138.

[36] N. Iwadate, M. Suginome, Org. Lett. 2009, 11, 1899-1902.

[37] H. Yoshida, M. Kimura, H. Tanaka, Y. Murashige, I. Kageyuki, I. Osaka, Chem. Commun. 2019, 55, 5420-5422.

[38] J. Szyling, A. Franczyk, K. Stefanowska, H. Maciejewski, J. Walkowiak, ACS Sustain. Chem. Eng. 2018, 6, 10980-10988.

[39] J. Szyling, A. Franczyk, K. Stefanowska, M. Klarek, H. Maciejewski, J. Walkowiak, ChemCatChem 2018, 10, 531-539.

[40] L. C. Moraes, R. C. Figueiredo, J. P. Espinós, F. Vattier, A. Franconetti, C. Jaime, B. Lacroix, J.
Rojo, P. Lara, S. Conejero, Nanoscale 2020, 12, 6821-6831.

[41] A. Leyva, X. Zhang, A. Corma, Chem. Commun. 2009, 4947-4949.

[42] H. Yoshida, I. Kageyuki, K. Takaki, Org. Lett. 2014, 16, 3512-3515.

[43] Y. Wang, R. Guan, P. Sivaguru, X. Cong, X. Bi, Org. Lett. 2019, 21, 4035-4038.

[44] R. Mamidala, V. K. Pandey, A. Rit, Chem. Commun. 2019, 55, 989-992.

[45] S. Pereira, M. Srebnik, Organometallics 1995, 14, 3127-3128.

[46] M. Birepinte, V. Liautard, L. Chabaud, M. Pucheault, Org. Lett. 2020, 22, 2838-2843.

[47] J. Cid, J. J. Carbõ, E. Fernández, Chem. Eur. J. 2012 18, 12794-12802.

[48] X. He, J. F. Hartwig, J. Am. Chem. Soc. 1996, 118, 1696-1702.

[49] J. Bhattacharjee, A. Harinath, K. Bano, T. K. Panda, ACS Omega 2020, 5, 1595-1606.

[50] A. Brzozowska, V. Zubar, R.-C. Ganardi, M. Rueping, Org. Lett. 2020, 22, 3765-3769.

[51] M. D. Greenhalgh, S. P. Thomas, Chem. Commun. 2013, 49, 11230-11232.

[52] M. Haberberger, S. Enthaler, Chem. Asian J. 2013, 8, 50-54.

[53] V. S. Rawat, B. Sreedhar, Synlett 2014, 25, 11321136.

[54] A. Khan, A. M. Asiri, S. A. Kosa, H. Garcia, A. Grirrane, J. Catal. 2015, 329, 401-412.

[55] M. Espinal-Viguri, C. R. Woof, R. L. Webster, Chem. Eur. J. 2016, 22, 11605-11608.

[56] K. Nakajima, T. Kato, Y. Nishibayashi, Org. Lett. 2017, 19, 4323-4326.

[57] A. Singh, S. Shafiei-Haghighi, C. R. Smith, D. K. Unruh, M. Findlater, Asian J. Org. Chem. 2020, 9, 416-420.

[58] F. Rami, F. Bächtle, B. Plietker, Catal. Sci. Technol. 2020, 10, 1492-1497.

[59] H. Ben-Daat, C. L. Rock, M. Flores, T. L. Groy, A. C. Bowman, R. J. Trovitch, Chem. Commun. 2017, 53, 7333-7336.

[60] H. L. Sang, C. Wu, G. G. D. Phua, S. Ge, ACS Catal. 2019, 9, 10109-10114.

[61] G. Zhang, S. Li, J. Wu, H. Zeng, Z. Mo, K. Davis, S. Zheng, Org. Chem. Front. 2019, 6, 3228-3233.

[62] I. D. Gridnev, N. Miyaura, A. Suzuki, Organometallics 1993, 12, 589-592.

[63] H. R. Kim, J. Yun, Chem. Commun. 2011, 47, 2943-2945.

[64] K. Semba, T. Fujihara, J. Terao, Y. Tsuji, Chem. Eur. J. 2012, 18, 4179-4184.

[65] W. J. Jang, B. Kang, J. H. Lee, Y. M. Choi, C. Kim, J. Yun, Org. Biomol. Chem. 2019, 17, 5249-5252.

[66] H. Tsai, M. Madasu, M. H. Huang, Chem. Eur. J. 2019, 25, 1300-1303.

[67] L. L. Baldassari, K. S. Santos, C. P. Ebersol, D. S. Lüdtke, A. V. Moro, Catal. Sci. Technol. 2020, 10, 7476-7480.

[68] I. M. de Oliveira, H. A. Esteves, M. P. Darbem, A. 
Sartorelli, T. C. Correra, A. F. Rodrigues-Oliveira, D. C. Pimenta, J. Zukerman-Schpector, F. Manarin, H. A. Stefani, Chem CatChem 2020, 12, 3545-3552.

[69] M. Zhong, Y. Gagne, T. O. Hope, X. Pannecoucke, M. Frenette, P. Jubault, T. Poisson, Angew. Chem. Int. Ed. 2021, 60, 14498-14503.

[70] A. L. Moure, R. G. Arrayás, D. J. Cárdenas, I. Alonso, J. C. Carretero, J. Am. Chem. Soc. 2012, 134, 7219-7222.

[71] J. K. Park, B. A. Ondrusek, D. T. McQuade, Org. Lett. 2012, 14, 4790-4793.

[72] J. Zhao, Z. Niu, H. Fu, Y. Li, Chem. Commun. 2014, 50, 2058-2060.

[73] G. Zhu, W. Kong, H. Feng, Z. Qian, J. Org. Chem. 2014, 79, 1786-1795.

[74] Y. D. Bidal, F. Lazreg, C. S. J. Cazin, ACS Catal. 2014, 4, 1564-1569.

[75] E. A. Romero, R. Jazzar, G. Bertrand, J. Organomet. Chem. 2017, 829, 11-13.

[76] X. Zeng, C. Gong, H. Guo, H. Xu, J. Zhang, J. Xie, New J. Chem. 2018, 42, 17346-17350.

[77] J. W. Hall, D. M. L. Unson, P. Brunel, L. R. Collins, M. K. Cybulski, M. F. Mahon, M. K. Whittlesey, Organometallics 2018, 37, 3102-3110.

[78] S. Mandal, S. Mandal, K. Geetharani, Chem. - An Asian J. 2019, 14, 4553-4556.

[79] S. Mandal, P. K. Verma, K. Geetharani, Chem. Commun. 2018, 54, 13690-13693.

[80] Y. Wu, C. Shan, J. Ying, J. Su, J. Zhu, L. L. Liu, Y. Zhao, Green Chem. 2017, 19, 4169-4175.

[81] C. M. Deng, Y. F. Ma, Y. M. Wen, ChemistrySelect 2018, 3, 1202-1204.

[82] D. Yan, X. Wu, J. Xiao, Z. Zhu, X. Xu, X. Bao, Y. Yao, Q. Shen, M. Xue, Org. Chem. Front. 2019, 6, 648-653.

[83] Z.-C. Wang, M. Wang, J. Gao, S.-L. Shi, Y. Xu, Org. Chem. Front. 2019, 6, 2949-2953.

[84] M. K. Bisai, S. Yadav, T. Das, K. Vanka, S. S. Sen, Chem. Commun. 2019, 55, 11711-11714.

[85] J. Li, M. Luo, X. Sheng, H. Hua, W. Yao, S. A. Pullarkat, L. Xu, M. Ma, Org. Chem. Front. 2018, 5, 3538-3547.

[86] M. Magre, B. Maity, A. Falconnet, L. Cavallo, M. Rueping, Angew. Chem. Int. Ed. 2019, 58, 70257029.

[87] Z. Yang, M. Zhong, X. Ma, K. Nijesh, S. De, P. Parameswaran, H. W. Roesky, J. Am. Chem. Soc. 2016, 138, 2548-2551.

[88] A. Bismuto, S. P. Thomas, M. J. Cowley, Angew. Chem. Int. Ed. 2016, 55, 15356-15359.

[89] D. Franz, L. Sirtl, A. Pöthig, S. Inoue, Z. Anorg. Allg. Chem. 2016, 642, 1245-1250.

[90] V. A. Pollard, M. Á. Fuentes, A. R. Kennedy, R. McLellan, R. E. Mulvey, Angew. Chem. Int. Ed. 2018, 57, 10651-10655.

[91] G. Zhang, J. Wu, H. Zeng, M. C. Neary, M. Devany, S. Zheng, P. A. Dub, ACS Catal. 2019, 9, 874-884.

[92] A. Harinath, I. Banerjee, J. Bhattacharjee, T. K. Panda, New J. Chem. 2019, 43, 10531-10536.

[93] A. K. Jaladi, H. Kim, J. H. Lee, W. K. Shin, H.
Hwang, D. K. An, New J. Chem. 2019, 43, 16524 16529.

[94] N. Sarkar, S. Bera, S. Nembenna, J. Org. Chem. 2020, 85, 4999-5009.

[95] F. Li, X. Bai, Y. Cai, H. Li, S. Q. Zhang, F. H. Liu, X. Hong, Y. Xu, S. L. Shi, Org. Process Res. Dev. 2019, 23, 1703-1708.

[96] H. E. Ho, N. Asao, Y. Yamamoto, T. Jin, Org. Lett. 2014, 16, 4670-4673.

[97] M. Fleige, J. Möbus, T. Vom Stein, F. Glorius, D. W. Stephan, Chem. Commun. 2016, 52, 10830 10833.

[98] J. R. Lawson, L. C. Wilkins, R. L. Melen, Chem. Eur. J. 2017, 23, 10997-11000.

[99] S. Chen, L. Yang, D. Yi, Q. Fu, Z. Zhang, W. Liang, Q. Zhang, J. Ji, W. Wei, RSC Adv. 2017, 7, 2607026073.

[100] M. Aelterman, M. Sayes, P. Jubault, T. Poisson, Chem. Eur. J. 2021, 27, 8277-8282.

[101] T. Ohmura, Y. Yamamoto, N. Miyaura, J. Am. Chem. Soc. 2000, 122, 4990-4991.

[102] J. Cid, J. J. Carbó, E. Fernández, Chem. Eur. J. 2012, 18, 1512-1521.

[103] C. Gunanathan, M. Hölscher, F. Pan, W. Leitner, J. Am. Chem. Soc. 2012, 134, 14349-14352.

[104] B. Sundararaju, A. Fürstner, Angew. Chem. Int. Ed. 2013, 52, 14050-14054.

[105] L.-J. Song, T. Wang, X. Zhang, L. W. Chung, Y.-D. Wu, ACS Catal. 2017, 7, 1361-1368.

[106] K. Yamamoto, Y. Mohara, Y. Mutoh, S. Saito, J. Am. Chem. Soc. 2019, 141, 17042-17047.

[107] L. E. Longobardi, A. Fürstner, Chem. Eur. J. 2019 , 25, 10063-10068.

[108] S. Xu, Y. Zhang, B. Li, S.-Y. Liu, J. Am. Chem. Soc. 2016, 138, 14566-14569.

[109] Y. Yang, J. Jiang, H. Yu, J. Shi, Chem. Eur. J. 2018, 24, 178-186.

[110] W. J. Jang, W. L. Lee, J. H. Moon, J. Y. Lee, J. Yun, Org. Lett. 2016, 18, 1390-1393.

[111] J. V. Obligacion, J. M. Neely, A. N. Yazdani, I. Pappas, P. J. Chirik, J. Am. Chem. Soc. 2015, 137, 5855-5858.

[112] N. Gorgas, L. G. Alves, B. Stöger, A. M. Martins, L. F. Veiros, K. Kirchner, J. Am. Chem. Soc. 2017, 139, 8130-8133.

[113] N. Gorgas, B. Stöger, L. F. Veiros, K. Kirchner, ACS Catal. 2018, 8, 7973-7982.

[114] S. Garhwal, N. Fridman, G. De Ruiter, Inorg. Chem. 2020, 59, 13817-13821.

[115] J. S. McGough, S. M. Butler, I. A. Cade, M. J. Ingleson, Chem. Sci. 2016, 7, 3384-3389.

[116] K. Nagao, A. Yamazaki, H. Ohmiya, M. Sawamura, Org. Lett. 2018, 20, 1861-1865.

[117] Y. Zi, F. Schömberg, F. Seifert, H. Görls, I. Vilotijevic, Org. Biomol. Chem. 2018, 16, 63416349.

[118] R. Fritzemeier, A. Gates, X. Guo, Z. Lin, W. L. Santos, J. Org. Chem. 2018, 83, 10436-10444.

[119] H. Jang, A. R. Zhugralin, Y. Lee, A. H. Hoveyda, J. Am. Chem. Soc. 2011, 133, 7859-7871. 
[120] A. L. Moure, P. Mauleón, R. G. Arrayás, J. C. Carretero, Org. Lett. 2013, 15, 2054-2057.

[121] H. Yoshida, Y. Takemoto, K. Takaki, Chem. Commun. 2014, 50, 8299-8302.

[122] J. S. Da Costa, R. K. Braun, P. A. Horn, D. S.
Lüdtke, A. V. Moro, RSC Adv. 2016, 6, 5993559938.

[123] T. A. Dibenedetto, A. M. Parsons, W. D. Jones, Organometallics 2019, 38, 3322-3326. 
